\title{
OPEN The histone replacement gene His4r is involved in heat stress induced chromatin rearrangement
}

\begin{abstract}
Anikó Faragó ${ }^{1,2}$, Adél Ürmösi ${ }^{1,2}$, Anita Farkas ${ }^{1,2}$ \& László Bodai ${ }^{1 凶}$
His4r is the only known variant of histone $\mathrm{H} 4$ in Drosophila. It is encoded by the His4r single-copy gene that is located outside of the histone gene cluster and expressed in a different pattern than $\mathrm{H} 4$, although the encoded polypeptides are identical. We generated a null mutant $\left(\mathrm{His}_{4} \mathrm{r}^{42}\right.$ ) which is homozygous viable and fertile without any apparent morphological defects. Heterozygous His $4 r^{\Delta 42}$ is a mild suppressor of position-effect variegation, suggesting that His4r has a role in the formation or maintenance of condensed chromatin. Under standard conditions loss of His4r has a modest effect on gene expression. Upon heat-stress the induction of the Heat shock protein (HSP) genes Hsp27 and Hsp68 is stronger in His $4 r^{442}$ mutants with concordantly increased survival rate. Analysis of chromatin accessibility after heat shock at a $\mathrm{Hsp} 27$ regulatory region showed less condensed chromatin in the absence of His4r while there was no difference at the gene body. Interestingly, preconditioning before heat shock led to increased chromatin accessibility, HSP gene transcription and survival rate in control flies while it did not cause notable changes in His4 ${ }^{\Delta 42}$. Thus, our results suggest that His4r might play a role in fine tuning chromatin structure at inducible gene promoters upon environmental stress conditions.
\end{abstract}

Packaging of chromosomal DNA in the nucleus is accomplished by the formation of chromatin structure which is a complex of DNA, RNA, and histone and non-histone proteins ${ }^{1,2}$. The basic repeating unit of chromatin is the nucleosome which consists of $147 \mathrm{bp}$ DNA wrapped around an octamer of core histones $\mathrm{H} 2 \mathrm{~A}, \mathrm{H} 2 \mathrm{~B}, \mathrm{H} 3 \mathrm{and}$ $\mathrm{H} 4^{1,3}$. The chromatin fiber can go through further condensation and form either loosely packed euchromatin or more condensed heterochromatin ${ }^{4}$. The chromatin structure hinders the accessibility of DNA to non-histone proteins that control replication, gene expression or DNA repair. In favor of these events chromatin is relaxed in a coordinated and organized way through chromatin remodeling or histone modifications ${ }^{5}$.

One way of chromatin remodeling is the replacement of nucleosomal histones by histone variants ${ }^{6}$. In higher eukaryotes replication dependent, so called canonical histone genes can be found in tandem copies in histone gene clusters that are mainly active during the $S$ phase of the cell cycle. Genes of canonical histones do not possess introns and their transcripts do not have poly(A) tails. Histone variants, on the other hand, are encoded by intron containing single-copy genes that express polyadenilated mRNA in nondividing, terminally differentiated tissues $^{7,8}$. All known histone variant genes encode proteins that differ from the canonical histones ${ }^{9}$, except for one, histone $\mathrm{H} 4$

In Drosophila a single-copy histone $\mathrm{H} 4$ variant gene was identified by Akhmanova et al. that encodes a protein with the same amino acid sequence as canonical H4 and was consequently named H4 replacement (CG3379, henceforth His4r) as due to their identity at the protein level His $4 \mathrm{r}$ is a replacement histone not a variant ${ }^{10}$. Unlike canonical histone $\mathrm{H} 4$ genes $\mathrm{His} 4 \mathrm{r}$ contains 2 introns and its transcript is polyadenilated ${ }^{10,11}$ and similarly to other histone variant genes ${ }^{12,13}$ it is localized outside of the histone gene cluster. According to high-throughput expression data His $4 r$ has a relatively uniform expression profile in all tissues and developmental stages ${ }^{14,15}$ suggesting that it serves functions utilized by all cell types and that its specific functions might be defined by post-transcriptional processes ${ }^{11}$.

Among closely related Drosophila species the gene structure of His4r is very similar with only minor differences in the nucleotide sequences encoding for identical proteins. This strong conservation led to the assumption that His4r, similarly to H3.3, might play a role in histone replacement ${ }^{11}$.

\footnotetext{
${ }^{1}$ Department of Biochemistry and Molecular Biology, Faculty of Science and Informatics, University of Szeged, Közép fasor 52, 6726 Szeged, Hungary. ${ }^{2}$ Doctoral School in Biology, Faculty of Science and Informatics, University of Szeged, 6726 Szeged, Hungary. ${ }^{\varpi}$ email: bodai@bio.u-szeged.hu
} 
A

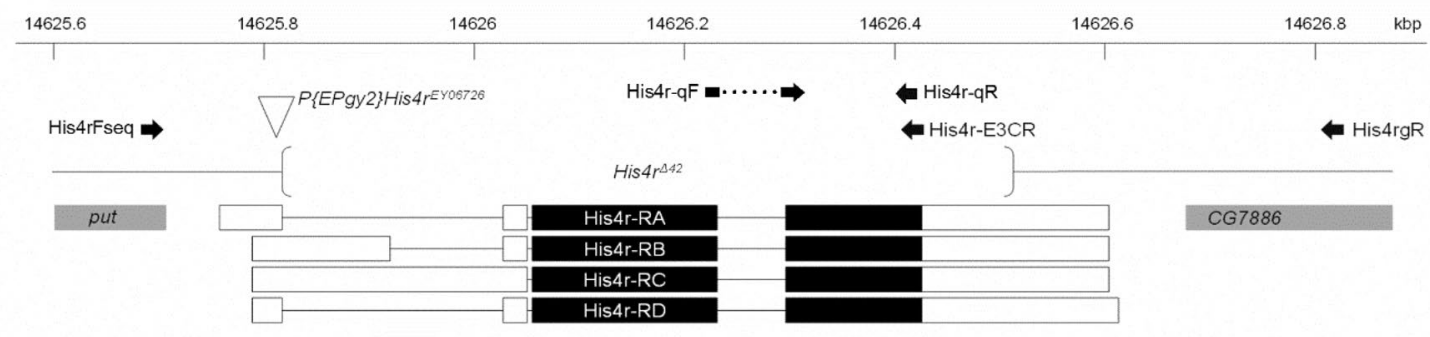

B

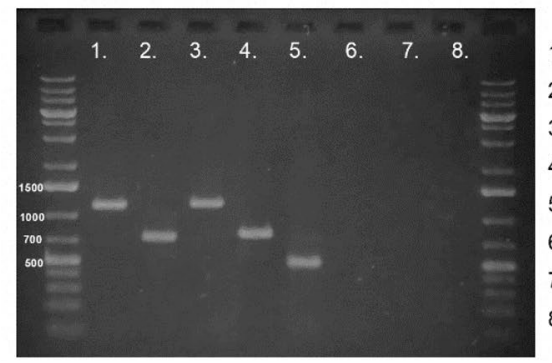

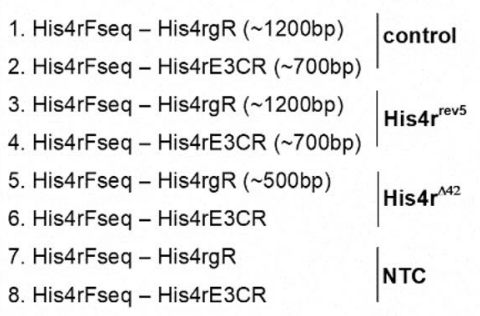

C

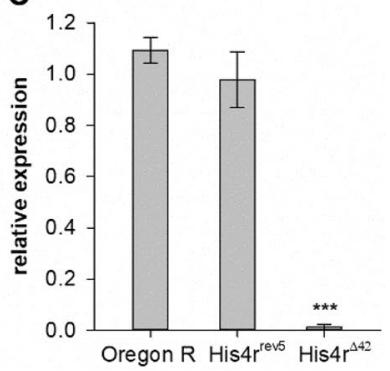

Figure 1. Generation and validation of the His $4 r^{\Delta 42}$ deletion mutant. (A) The genomic region of His $4 r$ on chromosome arm 3R with transcript models, primers and the extent of the deletion is shown. On the His4r transcript models coding sequences are represented by black bars, untranslated exonic regions by empty bars, and introns by solid lines. Adjacent ends of neighboring genes ( $p u t$ and CG7886) are shown with grey bars. Empty arrowhead indicates the position of the P\{EPgy2 $\}$ His $4 r^{E Y 06726}$ transposon in the His4r 5'-UTR, brackets show the breakpoints of the His $4 r^{\Delta 42}$ deletion. Position of PCR primers are marked by black arrows. (B) PCR reactions on homozygous His $4 r^{\Delta 42}$ template with His4rFseq and His4r-E3CR primers did not give a PCR product, with His4rFseq and His4rgR primers the length of the product was approximately 700 bp shorter than on wild-type control template. PCR reactions on His4rev5 templates resulted in amplicons similar to wildtype. (C) His4r transcript levels were similar in Oregon-R (wild-type) and in His $4 r^{\text {rev5 }}$ revertant flies $(\mathrm{P}>0.05)$, while in His $4 r^{\Delta 42}$ mutants His $4 r$ mRNA was not present $\left(\mathrm{P}=2.69077 \times 10^{-5}\right.$, t-test). The bars show the mean of normalized RT-qPCR measurements relative to a template dilution series, error bars represent standard error, $\mathrm{n}=3$.

To date there are only a few reports about $\mathrm{H} 4$ replacement histones in other species like H4V in Trypanosoma ${ }^{16}, \mathrm{H} 4 \mathrm{v}$ in Neurospora crassa ${ }^{17}$ or bovine H4-v. $1^{18}$. Although $\mathrm{H} 4$ replacement genes have been identified in several species no functional characterization has been reported and their functions are yet to be clarified.

To analyze Drosophila melanogaster His $4 r$ we generated a null mutant $\left(H i s 4 r^{\Delta 42}\right)$ by deleting the entire coding region of the His $4 r$ gene and performed phenotypic characterization assays. His $4 r^{\Delta 42}$ flies are homozygous viable and fertile, however, we observed mild sublethality in females. We performed position-effect variegation (PEV) and transcriptome analysis as His $4 \mathrm{r}$ being a histone protein is expected to have a role in chromatin organization and found that loss of His4r acts as a mild PEV suppressor and has a modest effect on gene expression. To analyze the role of His4r in an inducible gene expression setting we investigated transcriptional and chromatin accessibility changes of Heat shock protein (HSP) genes upon heat shock treatment and found that lack of His4r has effects similar to preconditioning: increased HSP transcription, increased chromatin accessibility of the Hsp 27 promoter, and improved survival. Thus, our results indicate that His4r plays a role in the formation of local chromatin structure at inducible gene promoters.

\section{Results}

Generation of the His $4 r^{\Delta 42}$ null allele. To be able to investigate in vivo functions of the Histone H4 replacement (His4r, CG3379) gene we generated null alleles by remobilization of the transposon inserted in the $5^{\prime}$-UTR of His4r at genomic position 3R:14,625,809 (Dm r6.15, FB2017_06) in the homozygous viable P\{EPgy2\}His$4 r^{E Y 06726}$ line. After remobilization of the P\{EPgy2\} element we selected revertants based on loss of the dominant mini-white marker gene and identified mutants in which deletions were generated as a consequence of imprecise transposon removal by screening revertants with PCR using primers straddling the His $4 r$ gene (Fig. 1A). The His4rFseq and His4rgR primers used are located -55 bp upstream and +211 bp downstream of the His $4 r$ locus [3R:14,625,755.0.14,626,614], respectively, and produce a 1162 bp PCR product on wild-type template. Among over 200 tested revertants we found a single candidate, His $4 r^{\Delta 42}$, which gave a shorter, approximately 500 bp long PCR amplicon (Fig. 1B, Supplementary Fig. S1). PCR reaction on homozygous His $4 r^{\Delta 42}$ template with His4rFseq and His4rE3CR primers, the latter of which is located at the $3^{\prime}$ end of His4 $r$ coding sequence, did not produce a PCR amplicon suggesting that the deletion removes the coding region of His $4 r$ (Fig. 1B). We verified these results and determined the extent of the deletion by capillary sequencing. In His $4 r^{\Delta 42}$ a 694 bp deletion extends from a breakpoint in the 5'-UTR $(3 R: 14,625,816)$ of His $4 r$ to one in the $3^{\prime}$-UTR $(3 R: 14,626,511)$ remov- 
ing the entire coding region but not affecting neighboring genes (Fig. 1A). The breakpoints enclose a 29 bp scar sequence containing remnants of the P $\{E P g y 2\}$ element (Supplementary Data S1).

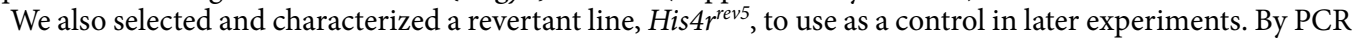
genotyping (Fig. 1B) and capillary sequencing we found that in this line the remobilized P\{EPgy2\} transposon did not cause alterations in the gene sequence except for a $40 \mathrm{bp}$ transposon derived scar sequence in the $5^{\prime}$-UTR straddled by 8 bp target site duplication (3R:14,625,809-14,625,816) (Supplementary Data S1). To determine whether the residual scar sequence affects the expression of His $4 r$ we compared His4r transcript levels in Oregon$R$ (wild-type), His $4 r^{\text {rev } 5}$ and His $4 r^{442}$ flies by reverse transcription quantitative PCR (RT-qPCR) and found that while His $4 r$ transcript was detected only at noise level in the deletion mutant its expression was not affected in the revertant strain (Fig. 1C).

Loss of His4r causes reduced viability but does not affect fertility. Similarly to the $P\{E P g y 2\} H i s-$ $4 r^{E Y 06726}$ parent strain the generated His $4 r^{\Delta 42}$ strain proved to be homozygous viable and fertile without any apparent morphological defects. To determine whether loss of His $4 r$ causes any phenotypic abnormalities or His $4 \mathrm{r}$ functions are redundant we performed tests to assess potential changes in fertility, viability and longevity. Previously it was reported that flies homozygous for the piggyBac induced insertion allele His $4 r^{L L 0551219}$ were sterile $^{20}$. To clear this contradiction we performed complementation tests. We crossed male or female w; His$4 r^{L L 05512} /$ His $4 r^{\Delta 42}$ heterozygotes with $w^{1118}$ flies and found that both crosses resulted in viable progeny (Supplementary Fig. S2). Thus, we conclude that the sterility of the His $4 r^{L L 05512}$ strain is probably caused by a background mutation. Next, we performed fecundity assays and found that the egg laying capacity of homozygous His $4 r^{\Delta 42}$ females $\left(15.3 \pm 0.99\right.$ eggs/female/day) was similar to that of His $4 r^{\text {rev }}$ control females $(15.04 \pm 1.2$ eggs/female/ day) (Fig. 2A). To test the viability of His $4 r^{\Delta 42}$ mutants we set up crosses to compare the eclosion rates of His $4 r^{\Delta 42}$ homozygous and heterozygous siblings. The number of homozygous His $4 r^{\Delta 42}$ offspring emerging from these crosses were lower than the number of heterozygous siblings (1321 vs 1940 , respectively, $\mathrm{P}<10^{-6}$, binomial test) (Fig. 2B), and the percentage of homo- and heterozygous offspring per vial $(40.6 \pm 0.6 \%$ and $59.4 \pm 0.6 \%$, respectively) significantly deviated from the expected $1: 1$ ratio $\left(\mathrm{P}=8 \times 10^{-32}, \mathrm{t}\right.$-test $)$. These findings suggest that the deletion of His $4 r$ causes a sublethal developmental defect. We also observed that among the homozygous $H i s 4 r^{\Delta 42}$ progeny the number of females was significantly lower than that of males (612 vs 709 , respectively, $\mathrm{P}=6.2 \times 10^{-4}$, binomial test) (Fig. $2 \mathrm{~B}$ ). To determine whether females are lost before or after pupariation we compared the male:female ratio of His $4 r^{\Delta 42}$ mutants and His $4 r^{\text {rev5 }}$ controls at different developmental stages. During the wandering L3 larval stage the average ratio of males and females in the His $4 r^{\text {rev } 5}$ control strain was very close to the expected $50-50 \%$ while in the case of His $4 r^{\Delta 42}$ it shifted to $59.4 \pm 1.6 \%$ males and $40.6 \pm 1.6 \%$ females $\left(\mathrm{P}=3 \times 10^{-10}\right.$, $\mathrm{t}$-test) (Fig. $\left.2 \mathrm{C}\right)$. When examining the pupation rates of the His $4 r^{\Delta 42}$ male or female larvae no significant loss could be observed compared to the controls (Fig. 2D). The eclosion rates of both His $4 r^{\Delta 42}$ male and female pupae were lower $(\mathrm{P}=0.01$ and $\mathrm{P}=0.02$, respectively, $\mathrm{t}$-test $)$ than that of the controls, however, there was no significant difference between the eclosion rates of the two genders (Fig. 2E). In conclusion, we could observe significant loss of $\mathrm{His}_{4} \mathrm{r}^{\Delta 42}$ individuals both during pupal development and during the development stages before pupation. The distorted male:female ratio of His $4 r^{\Delta 42}$ flies is resulted from the loss of females during embryonic or larval development but is not exacerbated during metamorphosis.

The expression of His4r decreases during aging but its loss does not affect lifespan. As besides mild sublethality we did not detect substantial developmental defects in $H i s 4 r^{\Delta 42}$ flies, we turned our attention to adult phenotypes. First, we characterized His4r gene expression during aging in wild-type $\left(w^{1118}\right)$ flies by measuring transcript levels at set time points using RT-qPCR. During the first 8 weeks of adult life we observed a gradual decrease in the expression of His $4 r$ which can be considered a silencing of gene activity with age $\left(\mathrm{P}=6.1004 \times 10^{-8}\right.$, One-Way ANOVA). During the first week the expression of His4r stabilized at around $80 \%$ of the level measured at the first day after eclosion from the pupal case, by weeks 4-6 it dropped to $50-60 \%$ and by the end of the second month it was as low as $12 \%$ of the initial level (Fig. 3A). Based on these expression data His $4 r$ transcription declines during the normal aging process. However, when performing longevity assays we did not detect major differences either in the time of $50 \%$ mortality rate (males: His $4 r^{r e v 5} 42.83$ days vs. His $4 r^{\Delta 42}$ 44.63 days; females: His $4 r^{\text {rev5 }} 54.9$ days vs. His $4 r^{442} 54.75$ days) or in maximum lifespan (males: His $4 r^{\text {rev5 }} 67$ days vs. His $4 r^{\Delta 42} 65$ days; females: His $4 r^{r e v 5} 79$ days vs. His $4 r^{\Delta 42} 78$ days) between the His $4 r$ mutant and the control strain (Fig. 3B).

Loss of His4r does not affect circadian activity. Recent studies revealed that transcription-permissive chromatin states are dynamically established in a circadian-time-specific manner, thus, chromatin remodeling appears to be a major regulatory mechanism in the circadian machinery ${ }^{21-23}$. Since His4r has been suggested to have a role in chromatin remodeling ${ }^{11}$ we were curious whether loss of His $4 r$ affects the circadian rhythm of flies and performed circadian activity measurements. Circadian rhythm of male Drosophila can be characterized by two activity peaks, one in the early hours of the morning and another at dusk, while they are less active in between, with nearly continuous inactivity at night ${ }^{24}$. We compared the daily activity of 1-week-old His $4 r^{\text {rev5 }}$ and $H i s 4 r^{\Delta 42}$ male Drosophila over a $24 \mathrm{~h}$ period of time. Our results show that the loss of His $4 r$ has no remarkable effect on the sleep pattern (Fig. 3C), total daily movement count (Fig. 3D) or the total amount of sleep (Fig. 3E), suggesting that His4r has no significant role in circadian related chromatin remodeling processes.

Loss of His4r has a mild effect on chromatin structure and transcription. As a histone replacement protein His4r was assumed to have a role in chromatin organization. To test the involvement of His4r in chromatin organization we performed position-effect variegation (PEV) analysis. When an euchromatic gene 
A

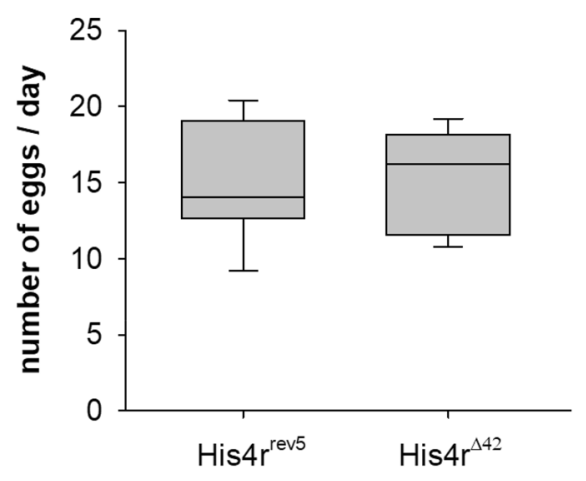

B

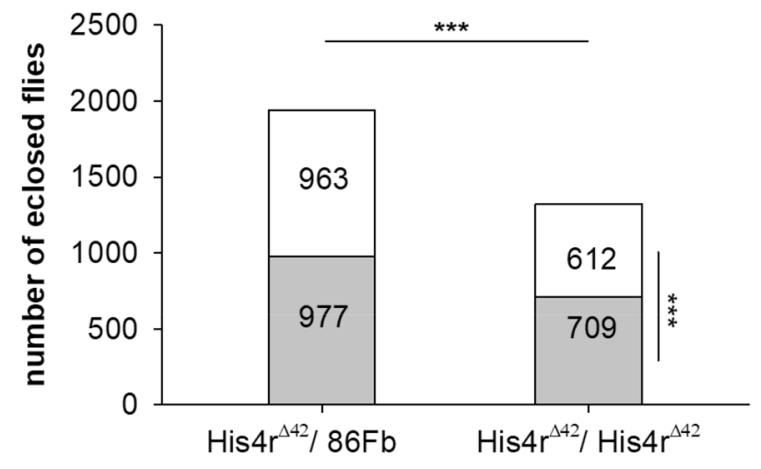

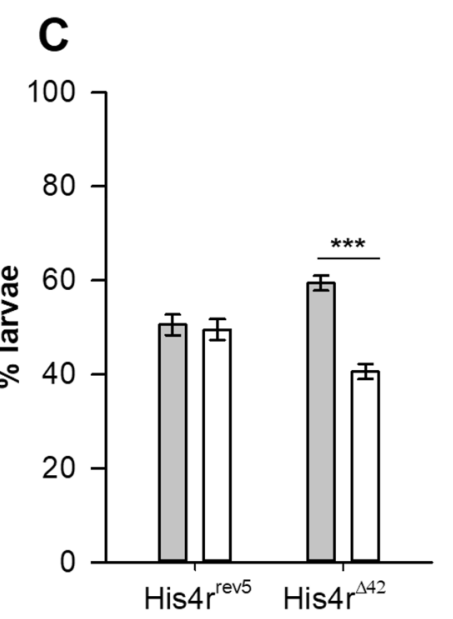
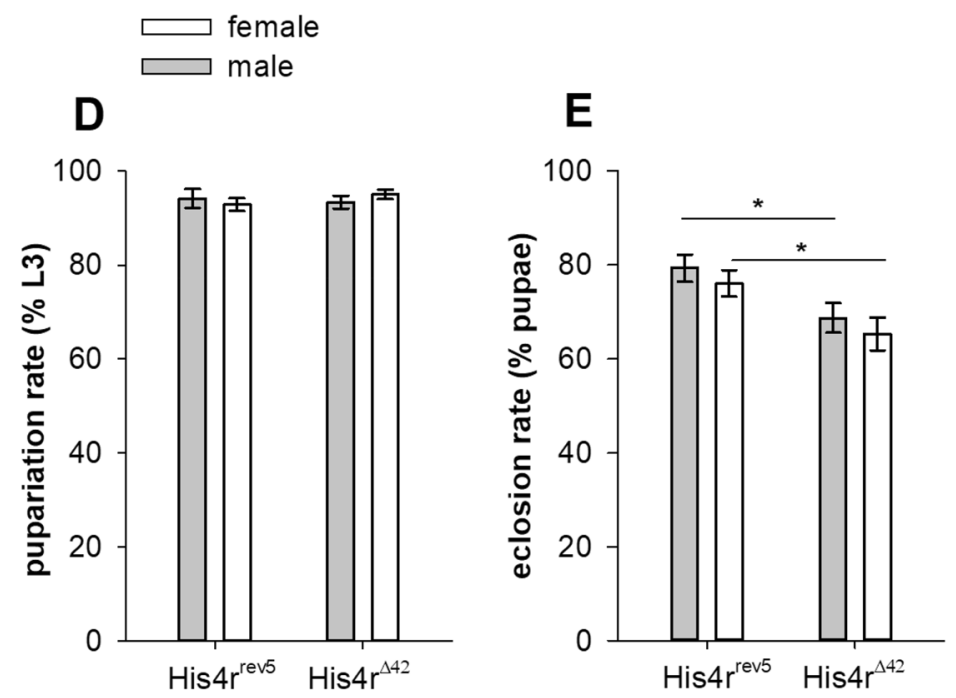

Figure 2. Loss of His $4 r$ reduces viability but does not affect fertility. (A) There was no significant difference in the fecundity of His $4 r^{\Delta 42}$ and His $4 r^{r e v 5}$ control females. Boxplot shows the distribution (first quartile, median, third quartile, and 10th and 90th percentiles as whiskers) of eggs laid by females daily, $n=5$ females in 10 vials per each genotype. (B) The number of eclosed homozygous $\operatorname{His} 4 r^{\Delta 42}$ flies was significantly lower $\left(\mathrm{P}<10^{-6}\right.$, binomial test) than that of heterozygous control siblings and among homozygous His $4 r^{\Delta 42}$ progeny the number of females was significantly lower than that of males $\left(\mathrm{P}=6.2 \times 10^{-4}\right.$, binomial test). (C) Among His4r ${ }^{\Delta 42}$ homozygous $\mathrm{L} 3$ larvae the ratio of females to males was significantly lower $\left(\mathrm{P}=3 \times 10^{-10}\right.$, $\mathrm{t}$-test $)$ than among His $4 r^{\text {rev5 }}$ controls. The bars show the percentage of male and female L3 larvae, error bars represent standard error, $\mathrm{n}=20$ vials per genotype. (D) The pupariation rate of $\mathrm{His} 4 \mathrm{r}^{\Delta 42}$ homozygotes did not change compared to His $4 r^{\text {rev } 5}$ controls. The bars show the percentage of pupariated L3 larvae, error bars represent standard error. (E) In $H_{i s} 4 r^{\Delta 4}$ homozygotes the eclosion rate of both male and female pupae were lower $(\mathrm{P}=0.01$ and $\mathrm{P}=0.02$, respectively, t-test) than that of the His $4 r^{r e v 5}$ controls. The bars show the percentage of eclosed pupae, error bars represent standard error. ${ }^{\star} \mathrm{P} \leq 0.05,{ }^{\star *} \mathrm{P}<10^{-3}$.

is juxtaposed with heterochromatin by rearrangement or transposition it causes its transcriptional silencing in some of the cells in a stochastic pattern. As this phenotype is the consequence of a change in the position of the gene in the genome, rather than a change in the gene itself, this phenomenon was named "position-effect variegation". For PEV analysis we applied the variegating phenotype of white-mottled $\left(w^{m 4}\right)$ allele, which is characterized by white spots in the otherwise red eyes. The whiter the eye the higher the rate of silencing ${ }^{25}$. Our results show that compared to His $4 r^{\text {rev }}$ controls heterozygous loss of His $4 r$ results in $\sim 15 \%$ higher eye pigment concentration both in males $\left(\mathrm{OD}_{485}\right.$ values: His $4 r^{\text {rev5 }} 0.2276 \pm 0.06$ and His $4 r^{\Delta 42} /+0.2623 \pm 0.05, \mathrm{P}=0.04452$, t-test) and in females $\left(\mathrm{OD}_{485}\right.$ values: His $4 r^{\text {rev5 }} 0.483 \pm 0.04$ and His $4 r^{44} /+0.55985 \pm 0.05, \mathrm{P}=0.00043$, t-test) (Fig. 4A). According to our findings His $4 r^{\Delta 42}$ acts as a mild dominant PEV suppressor, suggesting that His4r might have a role in the formation or maintenance of condensed chromatin structure.

Next, we aimed to assess whether loss of His $4 r$ that affects chromatin structure also influences transcriptional activity. For this we compared the transcriptome of 7-days-old adult $H i s 4 r^{\Delta 42}$ and $H i s 4 r^{\text {rev5 }}$ males by poly(A) RNA-sequencing (RNA-seq). We found that out of the 17,661 transcriptional units listed in Drosophila melanogaster gene annotation release r6.13 13,184 was expressed (using cut-off level of FPKM $\geq 1$ ) in at least one of the genotypes, 12,572 was expressed in both genotypes, and 11,207 had sequencing read counts and distribution suitable for statistical testing. We found that the transcriptome profile of His $4 r^{\Delta 42}$ flies was very similar to that of the His $4 r^{\text {rev } 5}$ revertant control with high correlation $(r=0.995)$ and median transcript levels of 7.915 FPKM 

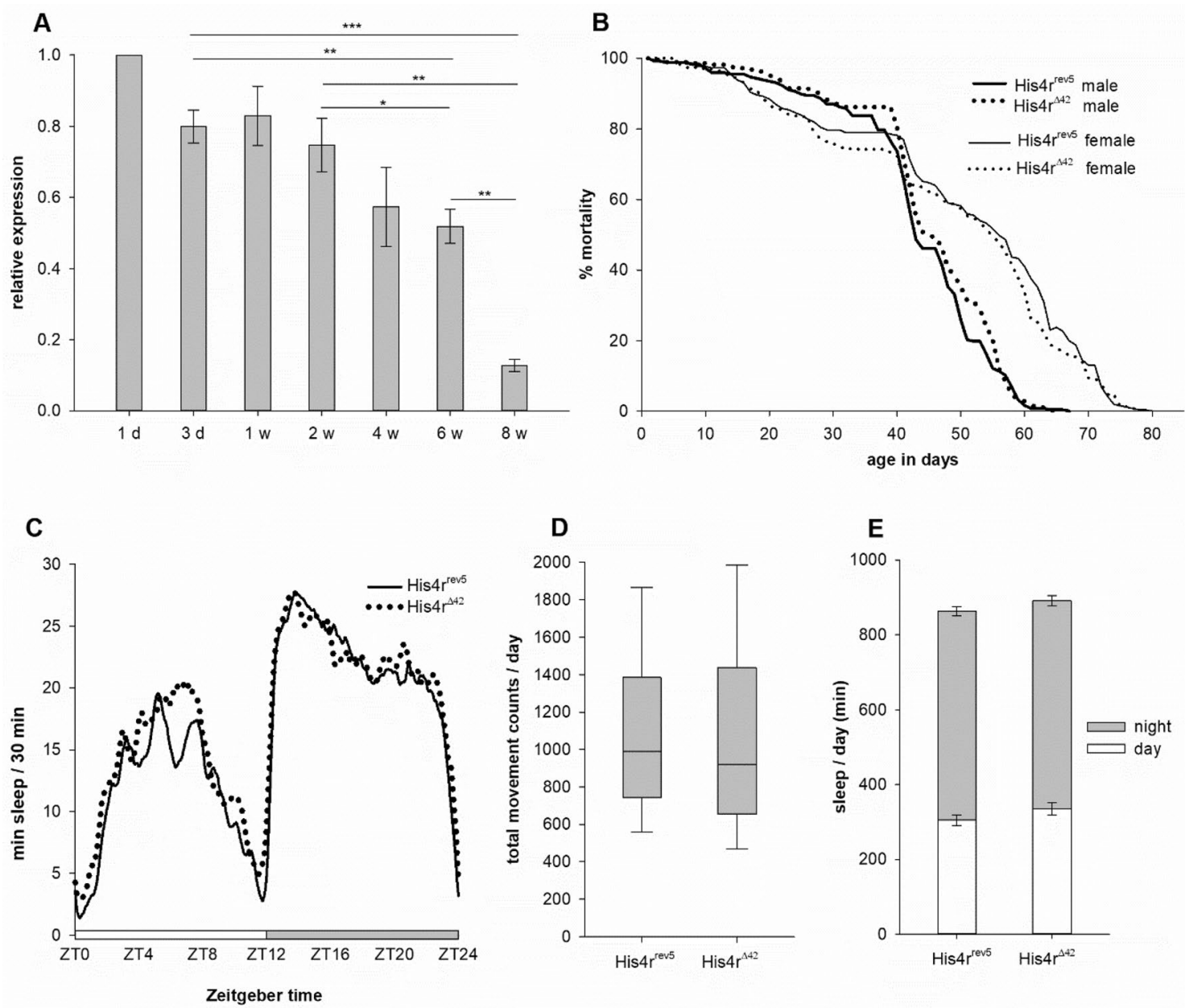

Figure 3. His $4 r$ is down-regulated with age but its loss does not affect longevity or circadian activity. (A) During the first 8 weeks of adult life His $4 r$ expression gradually decreased in male heads. Bars show the mean of normalized RT-qPCR measurements relative to a template dilution series, error bars represent standard error $(\mathrm{n} \geq 4) .{ }^{*} \mathrm{P}<0.05,{ }^{* * \mathrm{P}}<10^{-2},{ }^{* * *} \mathrm{P}<10^{-3}$ (OWA with Tukey HSD post-hoc test). (B) The longevity of homozygous His $4 r^{442}$ males and females was similar to that of their His $4 r^{r e v 5}$ control counterparts. The graph shows the percent of survivors as function of age ( $\mathrm{n} \geq 240$ in each category). (C) No notable difference could be observed between the sleep patterns of homozygous His $4 r^{\Delta 42}(\mathrm{n}=93)$ and His4rev5 $(\mathrm{n}=95)$ control males. (D) The daily movement of homozygous His $4 r^{\Delta 42}$ and His $4 r^{\text {rev } 5}$ control males was also similar. Boxplot shows the distribution (first quartile, median, third quartile, and 10th and 90th percentiles as whiskers) of movement counts of individual flies per day. (E) The total amount of time that homozygous $\mathrm{His}_{4} \mathrm{r}^{42}$ and His $4 r^{\text {rev5 }}$ control males spent asleep was also similar both daytime and nighttime.

(Fragments Per Kilobase of transcript per Million mapped reads) in the case of His4r mutants and 7.826 FPKM in the case of the revertants. In His $4 r$ mutants the transcript levels of 101 genes $(0.9 \%$ of statistically tested, $0.57 \%$ of total) was significantly (false discovery rate $\mathrm{q}<0.05$ ) altered: 67 was up-regulated while 34 was down-regulated (Fig. 4B). Of note, His4r was highly expressed in $\mathrm{His}_{4} \mathrm{r}^{\text {rev5 }}(\mathrm{FPKM}=336.95)$ while it was below the expression cut-off $(\mathrm{FPKM}=0.46)$ in $H i s 4 r^{\Delta 42}$, functionally validating both the revertant and the deletion mutant lines. Based on Gene Onthology analysis most up-regulated genes are associated with GO Biological Process terms of cellular process (GO:0009987), metabolic process (GO:0008152) or localization (GO:0051179) (Supplementary Fig. S3), while most down-regulated genes are associated with terms cellular process (GO:0009987), multicellular organismal process (GO:0032501) or metabolic process (GO:0008152) (Supplementary Fig. S4). The most dysregulated genes both among the up-regulated and down-regulated ones are associated with GO Molecular Function categories catalytic activity (GO:0003824) and binding (GO:0005488) (Supplementary Figs. S5 and S6). However, no molecular function or biological process terms were overrepresented in these groups at a statistically significant level.

His $4 r^{\Delta 42}$ flies show increased heat-stress resistance with stronger induction of heat shock protein genes and less condensed chromatin at the Hsp27 promoter. Next, we aimed to test whether His4r plays a role in the response to environmental stress. For this, we chose to analyze response to heat-stress, a well characterized paradigm in chromatin dynamics studies. First, we exposed 3-5 days old His $4 r^{\Delta 42}$ and His4r- 
A

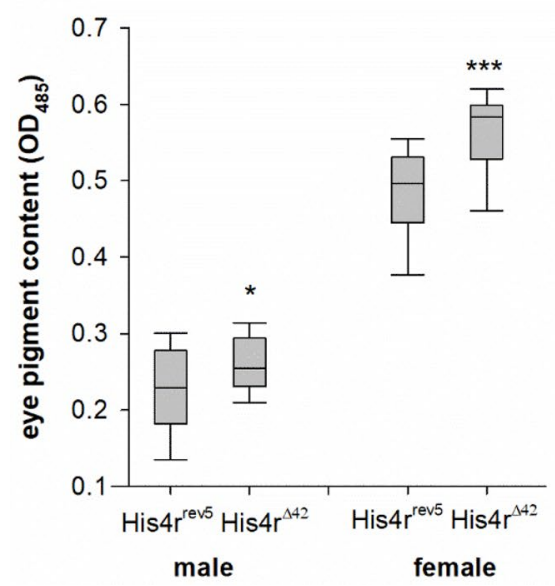

B

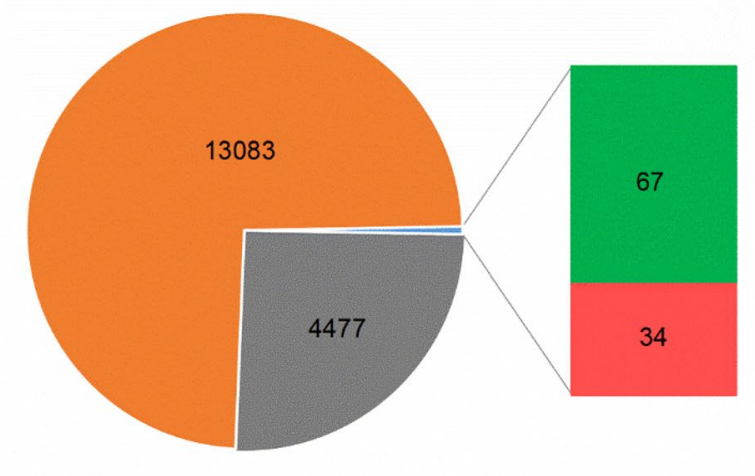

not expressed $n$ not changed $\square$ up-regulated down-regulated

Figure 4. Loss of His4r has mild effect on PEV and transcription. (A) Heterozygous loss of His4r results in mild but significant suppression of position-effect variegation of $w^{m 4 h}$ in both genders. Boxplot shows the distribution (first quartile, median, third quartile, and 10th and 90th percentiles as whiskers) of eye pigment absorbance values measured at $485 \mathrm{~nm}$. ${ }^{\star} \mathrm{P}<0.05,{ }^{\star * \star} \mathrm{P}<10^{-3}$ (t-test, $\mathrm{n}=20$ ). (B) Loss of His $4 r$ has a modest effect on transcriptional activity. Out of the 13,184 genes that were transcriptionally active (FPKM $\geq 1)$ in males only 101 showed altered expression levels in His $4 r^{\mathrm{S4}}$ homozygotes compared to His $4 r^{\text {rev5 }}$ controls: 67 were up- while 34 were down-regulated.

rev5 flies to $37^{\circ} \mathrm{C}$ heat shock for $0-5 \mathrm{~h}$ periods and determined their survival rates after specific lengths of heatstress $(0,1,2,3,3.5,4,4.5$, and $5 \mathrm{~h})$. We found that flies started to perish after $3 \mathrm{~h}$ of heat shock treatment and by $5 \mathrm{~h}$ only $20 \%$ was alive (Fig. $5 \mathrm{~A}, \mathrm{~B})$. The survival rates of both $H i s 4 r^{442}$ males $\left(\mathrm{P}<1.0 \times 10^{-10}\right.$, log-rank test) and females $\left(\mathrm{P}=4.4 \times 10^{-8}\right.$, log-rank test) was higher compared to the His $4 r^{\text {rev } 5}$ controls after $3.5 \mathrm{~h}$ of heat shock (Fig. 5A,B). Next, we tested the effect of preconditioning for $30 \mathrm{~min}$ at $34^{\circ} \mathrm{C}$ for 2 consecutive days prior to heat shock treatment at $37^{\circ} \mathrm{C}$ on the survival of 3-5 days old flies. We found that in these experiments flies started to perish after $3.5 \mathrm{~h}$ of $37^{\circ} \mathrm{C}$ heat shock and by $5 \mathrm{~h}$ of treatment only $20 \%$ percent was alive. Interestingly, after preconditioning there was no significant difference between the survival rates of His $4 r^{\Delta 42}$ and His $4 r^{\text {rev5 }}$ control flies, both groups had survival rates similar to that of $H i s 4 r^{\Delta 42}$ without preconditioning (Fig. 5C,D). We also performed the heat shock response experiment with 1-month-old flies that were exposed to $37^{\circ} \mathrm{C}$ heat shock for 0-120 min in $15 \mathrm{~min}$ intervals. These older flies were more sensitive to heat-stress, they started to perish after $1 \mathrm{~h}$ of $37^{\circ} \mathrm{C}$ heat shock treatment and by $2 \mathrm{~h}$ of treatment only $\sim 10-20 \%$ percent was alive both in the case of $H i s 4 r^{\Delta 42}$ and His $4 r^{\text {rev5 }}$ control males and females. The survival of both $H i s 4 r^{\Delta 42}$ males $(\mathrm{P}=0.009, \log$-rank test $)$ and females $(\mathrm{P}=0.0114$, log-rank test $)$ was only slightly better than the survival of corresponding control flies (Fig. 5E,F).

We hypothesized that increased heat-stress resistance in His $4 r$ mutants might be the consequence of increased expression of Heat shock protein (HSP) genes due to altered chromatin structure at regulatory elements. For testing we selected representative genes from each HSP family (Hsp27, Hsp60A, Hsp68 and Hsp83) that showed the highest expression upon heat shock according to modENCODE data ${ }^{14}$. We measured gene expression before heat shock and $0,0.5,1,1.5,2,3$, or $4 \mathrm{~h}$ after a $1 \mathrm{~h}$ long $37^{\circ} \mathrm{C}$ heat shock treatment by RT-qPCR and detected dramatic increase in the transcript levels of Hsp27, Hsp68 and Hsp83 after heat shock $(\mathrm{P}<0.001$ in each case both in $\mathrm{His}_{4} r^{42}$ and His $4 r^{\text {rev5 }}$ flies, Kruskal-Wallis Test) that gradually decreased during the post-heat shock incubation period (Fig. 6A,C,D), while Hsp60A showed a much milder (statistically not significant) response (Fig. 6B). We found that the induction of Hsp27 (Fig. 6A) and Hsp68 (Fig. 6C) was significantly stronger in His4r ${ }^{\Delta 42}$ than in control flies, while the induction of $H s p 83$ (Fig. 6D) was not significantly different between the two genotypes. It is worth to note that despite stronger gene induction in $H i s 4 r^{442}$ flies the passing off of transcript levels showed similar patterns in the two genotypes and evened up after $1.5 \mathrm{~h}$ in the case of $H s p 68$ and after $2 \mathrm{~h}$ in the case of $H s p 27$. We were interested to know whether the differences in the induction of HSP genes are cancelled out after preconditioning similarly to what we had observed in the case of post-heat shock survival rates. Therefore, we repeated the previous experiment after preconditioning flies for 2 days at $34^{\circ} \mathrm{C}$ for 30 min daily. After $37^{\circ} \mathrm{C}$ heat shock treatment the induction of HSP genes in $\mathrm{His}_{4} \mathrm{r}^{42}$ flies was very similar to the levels we measured without preconditioning. However, in $\mathrm{His}_{4} \mathrm{r}^{\text {rev } 5}$ controls the induction of HSP genes was more pronounced when the flies were preconditioned. Consequently, when preconditioning was applied HSP gene expression levels of His $4 r^{\Delta 42}$ and His $4 r^{\text {rev } 5}$ control flies became alike and reached similar elevated levels to what we had observed in His $4 r^{442}$ without preconditioning (Fig. 6E-H). Thus, preconditioning cancels out the differences both in survival rates and HSP transcript levels between His4r mutant and control flies.

Next, to test our hypothesis that His4r plays a role in the regulation of HSP genes by influencing local chromatin structure we performed chromatin accessibility assays. We analyzed the accessibility of the regulatory region containing three heat shock elements (HSE) upstream of the $H s p 27$ promoter $^{26}$ and the accessibility of the coding sequence of the gene by performing qPCR on MNase digested chromatin templates prepared from flies treated as 


\section{A}

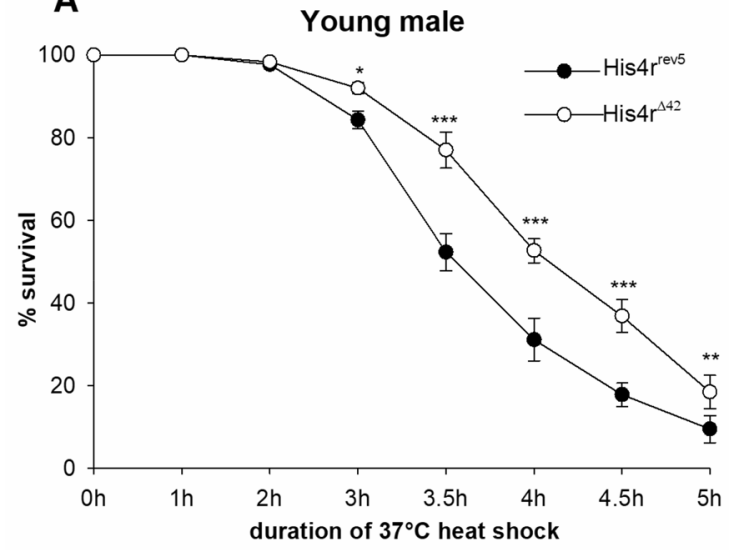

C

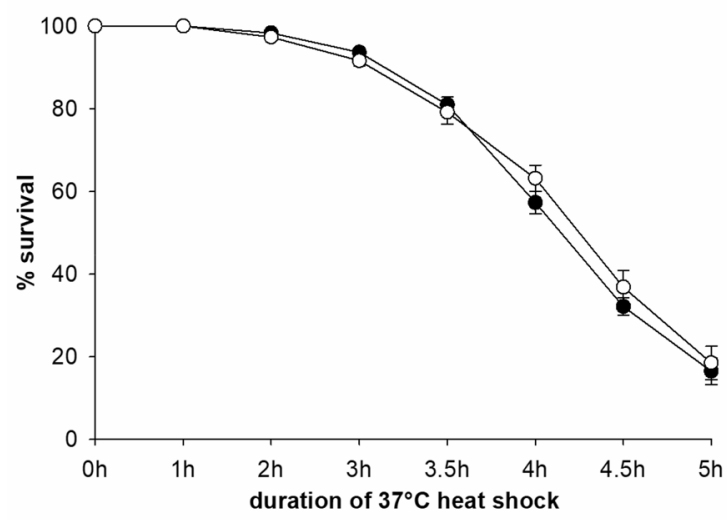

\section{E}

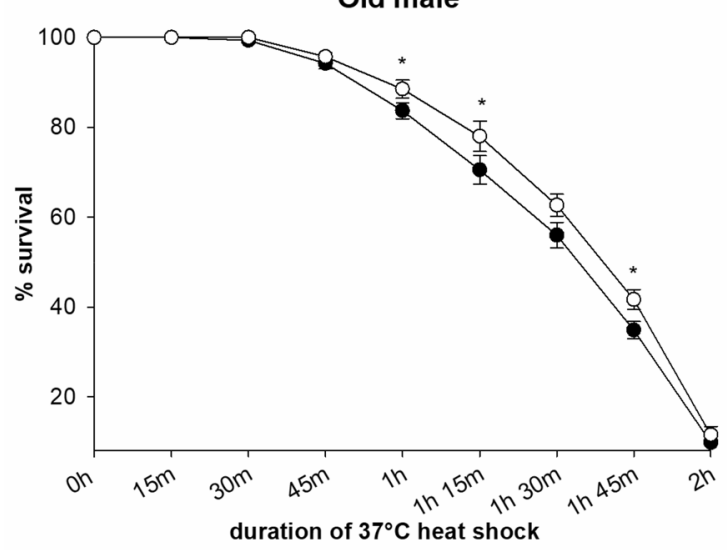

B

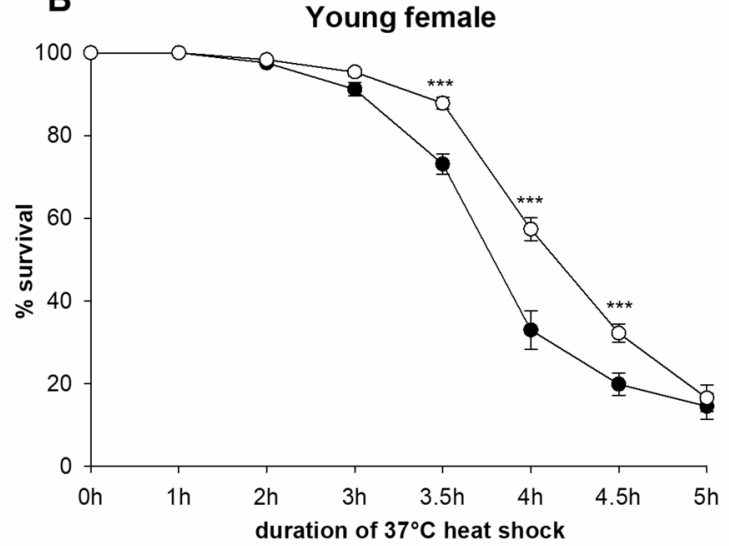

D Preconditioned young female

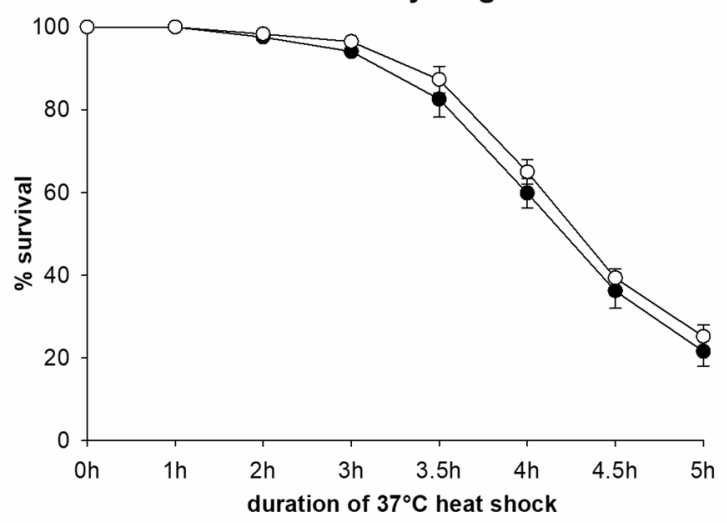

F

Old female

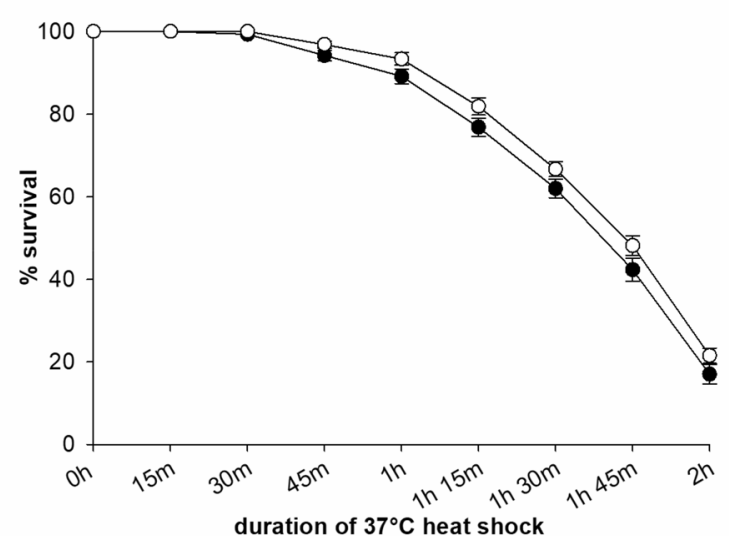

Figure 5. Loss of His $4 r$ increases heat-stress tolerance. Both 3-5 days old $H i s 4 r^{42}$ males (A) and females (B) showed increased heat-stress tolerance compared to revertant controls. The survival rate of $H i s 4 r^{\Delta 42}$ males was significantly higher than controls after $3.5-5 \mathrm{~h}$ of heat-stress at $37^{\circ} \mathrm{C}$, while the survival rate of $\mathrm{His}^{\circ} \mathrm{r}^{\Delta 42}$ females was higher than controls after $3.5-4.5 \mathrm{~h}$ of heat-stress. After preconditioning for $30 \mathrm{~min}$ at $34^{\circ} \mathrm{C}$ on two preceding days there was no difference between the survival rates of 3-5 days old His $4 r^{\Delta 42}$ males (C) or females (D) and corresponding controls after heat-stress at $37^{\circ} \mathrm{C} .30$ days old males (E) and females (F) showed reduced heat-stress tolerance compared to younger flies $(\mathbf{A}, \mathbf{B})$, and homozygous loss of His $4 r$ had only a minor effect on survival following heat shock (male: $\mathrm{P}=0.009$; female: $\mathrm{P}=0.0114$, log-rank test). The graph shows the average survival rate, error bars show standard error, $\mathrm{n}=30$ vials $\left(20\right.$ flies/vial). ${ }^{\star} \mathrm{P}<0.05,{ }^{* *} \mathrm{P}<10^{-2},{ }^{* *} \mathrm{P}<10^{-3}$, MannWhitney U test. 

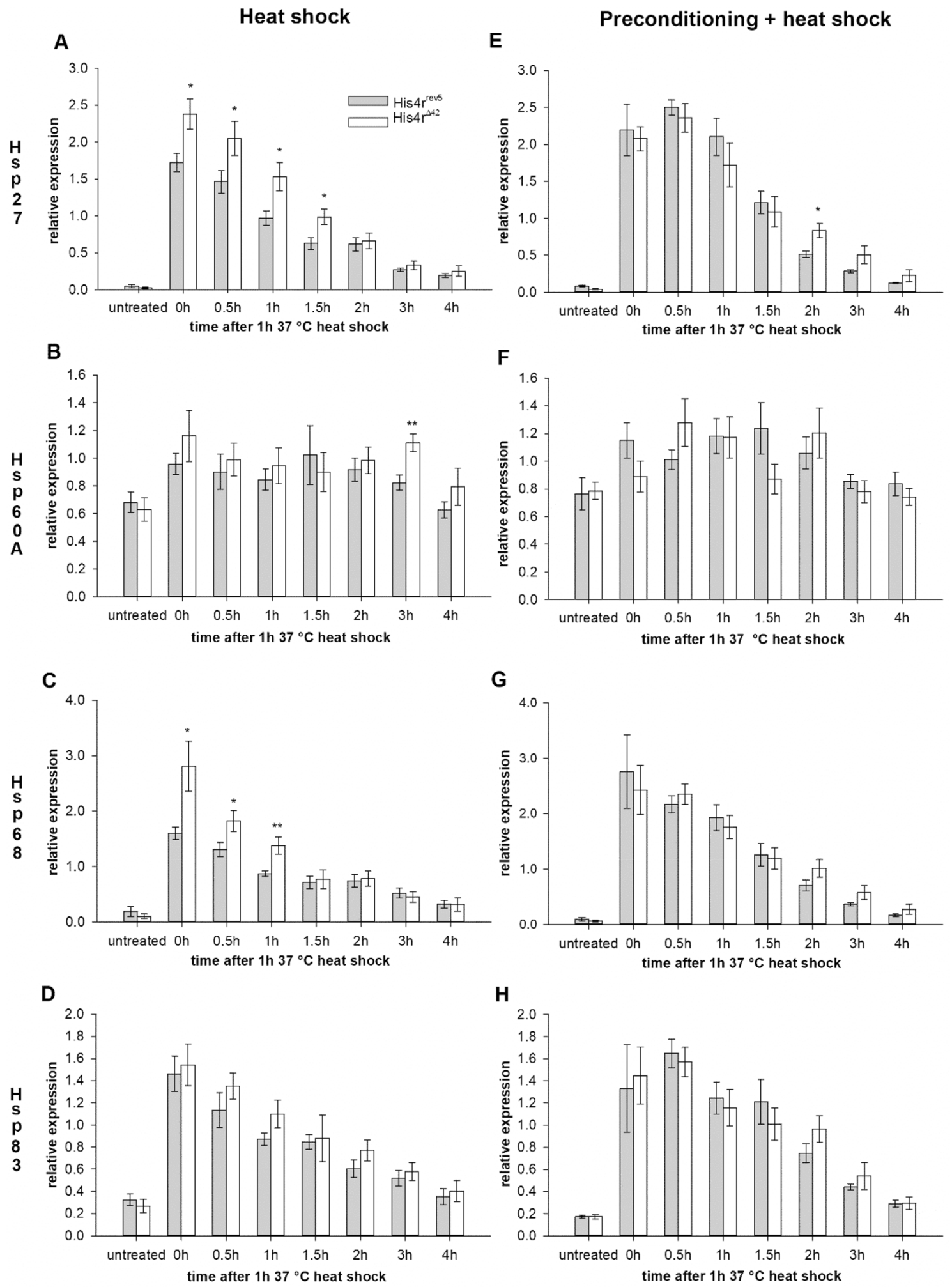

Figure 6. His4r affects HSP gene induction after heat shock. The transcript levels of $H s p 27(\mathbf{A}), H s p 68$ (C) and Hsp83 (D) HSP genes showed dramatic increase after $37^{\circ} \mathrm{C}$ heat shock $(\mathrm{P}<0.001$, Kruskal-Wallis Test) both in His4r mutants (white) and controls (grey) that gradually decreased during the post-heat shock incubation period, while there was no change in the expression of Hsp60A (B). However, following heat shock the transcript levels of Hsp27 (A) and Hsp68 (C) were significantly higher in His4r mutants than in controls. (E-H) After preconditioning at $34^{\circ} \mathrm{C}$ the expression of HSP genes in His $4 r^{r e v 5}$ controls reaches the same elevated levels as in $H i s 4 r^{\Delta 42}$ flies. Asterisks mark significant differences between $H i s 4 r^{\Delta 42}$ and His $4 r^{r e v 5}$ samples, ${ }^{\star} \mathrm{P}<0.05,{ }^{* *} \mathrm{P}<10^{-2}$, Mann-Whitney U test. 


\section{Heat shock}

A
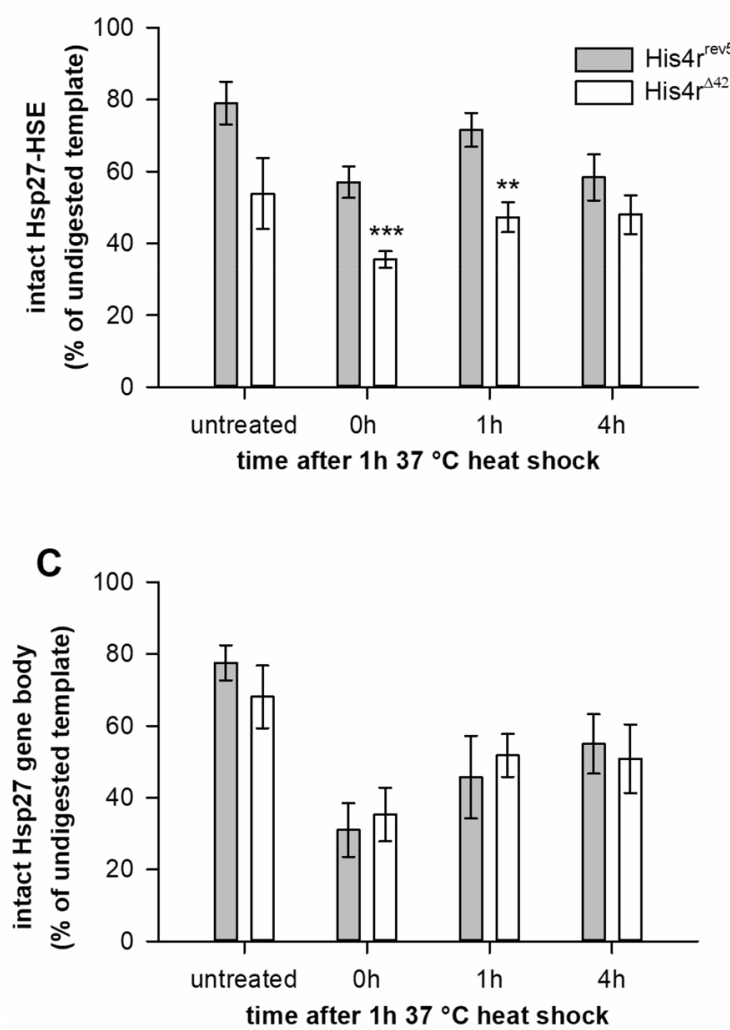

Preconditioning + heat shock

B
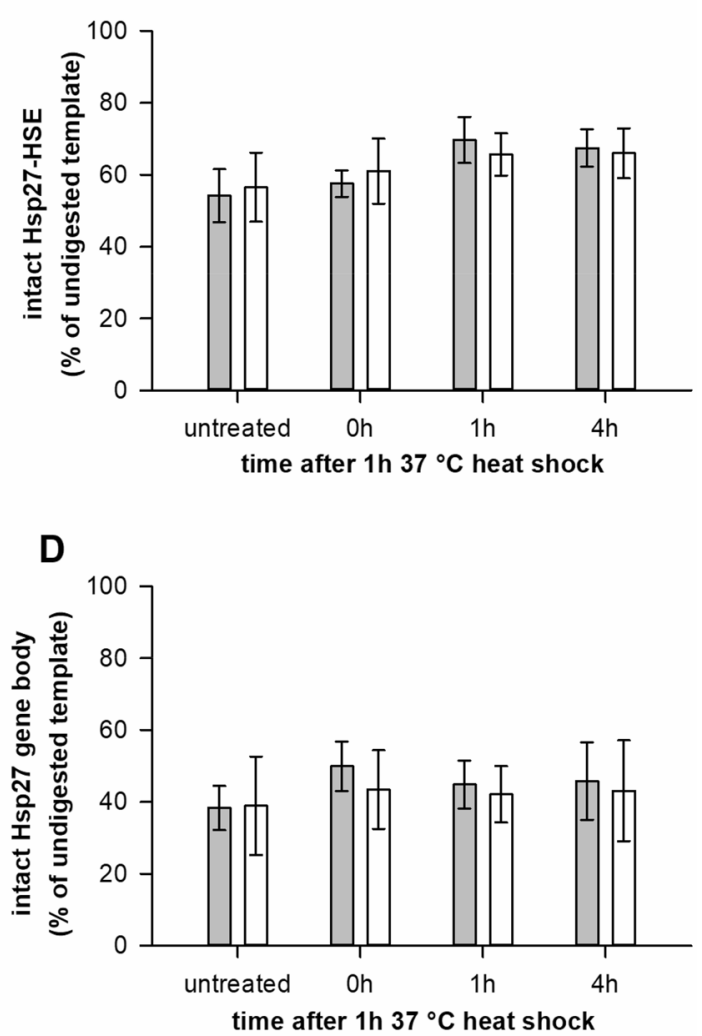

Figure 7. His4r is a component of chromatin at the HSE containing regulatory region of Hsp27. (A) In His4r mutants (white) lower amount of intact Hsp27-HSE was present after MNase digestion even without heat shock treatment compared to the controls, although this difference was not significant. Following heat shock significantly less PCR products were detected in $H i s 4 r^{\Delta 42}$ flies than in controls indicating more open chromatin.in the absence of His4r. (B) After preconditioning for 2 days in the controls the amount of intact Hsp27-HSE was reduced to the levels of His4r mutants cancelling out the difference between the two genotypes, furthermore, heat shock did not result in further chromatin opening. Chromatin accessibility at the gene body of Hsp27 in heat shock experiments without preconditioning (C) or with preconditioning (D) did not differ between His4r mutants and controls. ${ }^{* *} \mathrm{P}<10^{-2},{ }^{* *} \mathrm{P}<10^{-3}$, t-test.

in the previous experiments. In these experiments loss of nucleosomes allows the access of MNase to DNA that leads to less PCR products while the presence of intact nucleosomes protect DNA from MNase digestion and leads to more PCR products. $37^{\circ} \mathrm{C}$ heat shock induced loss of nucleosomes both at the HSE containing regulatory region (His $4 r^{\text {rev5 }} \mathrm{P}=0.01199$; His $4 r^{\Delta 42} \mathrm{P}=0.0375$, t-test) (Fig. 7A) and at the gene body (His $4 r^{\text {rev } 5} \mathrm{P}=0.00098$; His $4 r^{\Delta 42} \mathrm{P}=0.02244$, t-test) (Fig. 7C) that was detected by lower amount of intact DNA sequences after MNase treatment. In His $4 r^{\Delta 42}$ samples we measured lower amount of intact Hsp27-HSE elements after MNase digestion than in His $4 r^{\text {rev5 }}$ controls even before heat shock treatment, however this difference was not significant $(\mathrm{P}=0.07714$, t-test) (Fig. 7A). Immediately after the $1-\mathrm{h} 37^{\circ} \mathrm{C}$ heat shock treatment significantly less PCR product was detected in His $4 r^{\Delta 42}$ flies compared to the His $4 r^{\text {rev5 }}$ controls $(\mathrm{P}=0.00053$, t-test) (Fig. $7 \mathrm{~A})$ indicating that the chromatin structure of the Hsp27-HSE site was more accessible in the mutant. The amount of intact Hsp27-HSE templates in His $4 r^{\Delta 42}$ remains significantly lower $(\mathrm{P}=0.0022$, $\mathrm{t}$-test $)$ than in controls $1 \mathrm{~h}$ after the heat shock (Fig. 7A), however, this difference levels off $4 \mathrm{~h}$ after heat shock treatment (Fig. 7A). When we preconditioned His $4 r^{442}$ and His $4 r^{\text {rev } 5}$ flies for 2 days at $34^{\circ} \mathrm{C}$ we observed similar quantities of Hsp27-HSE specific PCR amplicons before heat shock in both genotypes (Fig. 7B) as in $\mathrm{His}_{4} \mathrm{r}^{\Delta 42}$ flies without preconditioning (Fig. 7A). This result indicates that preconditioning relaxed chromatin at the HSEs in the control while did not have an effect in the His4r mutant. Furthermore, preconditioning before heat shock treatment abolished the difference in the amount of intact HSP27HSE elements between His $4 r^{\Delta 42}$ and His $4 r^{r e v 5}$ controls at all inspected time points (Fig. 7B) similarly to what we had observed previously in the case of survival rates and HSP gene expression.

To investigate whether the effect of lack of His $4 \mathrm{r}$ is specific for the regulatory region of $H s p 27$ or also influences chromatin accessibility at the gene body we performed qPCRs on the same samples using Hsp27 coding sequence specific primers. Our results show that in heat shock experiments there is no difference in the accessibility of chromatin at the Hsp27 gene body between His $4 r^{\Delta 42}$ mutants and His $4 r^{r e v 5}$ controls neither without (Fig. 7C) or with preconditioning (Fig. 7D). This suggests that while His4r is a significant component of chromatin at the regulatory region of $H s p 27$ it does not play an important role at the gene body. 


\section{Discussion}

The packaging of DNA into chromatin structure presents a barrier to nuclear processes like replication, gene expression or DNA repair. One way of loosening condensed chromatin structure or altering chromatin state is through the replacement of canonical nucleosomal core histones with histone variant proteins ${ }^{5}$. Most histone variants can be associated with specific chromatin states or chromatin related processes, for example H2A.X is involved in DNA double-strand break repair, while H3.3 is deposited to transcribed genes ${ }^{6}$. In higher eukaryotes variants of all core histones but $\mathrm{H} 4$ exist. Histone $\mathrm{H} 4$ is a slowly evolving protein which shows divergence in some protozoan taxa but sequence variants in higher eukaryotes have not been identified yet ${ }^{27}$. In the Drosophila genus a single-copy gene variant of $\mathrm{H} 4, \mathrm{His} 4 r$, exists that is highly conserved in all 14 Drosophila species analyzed ${ }^{11}$. The amino acid sequence of His4r proteins is identical to that of $\mathrm{H} 4$ in most Drosophila species including $D$. melanogaster making His $4 \mathrm{r}$ rather a replacement histone than a variant ${ }^{10,11}$. However, His $4 r$ genes have different regulation and gene structure than canonical, replication-dependent $H 4$ genes located in the histone gene cluster $^{10,11}$. These features suggest that His4r might play a conserved role in chromatin remodeling but its specific functions must depend on the regulation of its expression.

In the His $4 r^{\Delta 42}$ mutant we generated the entire coding region is deleted making it an amorphic (genetic null) allele. Similarly to carriers of loss of function mutations of histone $\mathrm{H} 3$ variants His3.3A or His3.3 ${ }^{28,29}$ homozy-

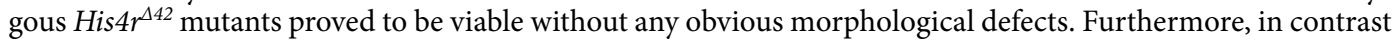
to the sterility caused by complete loss of $\mathrm{H} 3.3$ in $\mathrm{H3} .3 \mathrm{~B}$; $\mathrm{H3} .3 \mathrm{~A}$ double mutants ${ }^{28,29}$ both genders are fertile in the absence of His4r. We observed mild ( 32\%) sublethality of His $4 r$ mutant animals that occurred in part before pupariation and in part during metamorphosis. Remarkably, the viability of $H_{i s} 4 r^{\Delta 42}$ females was lower than that of male siblings and this disproportional loss of females occurred before pupariation during the embryonic and/or larval developmental stages. There are at least two sex specific processes that might be responsible for this phenomenon: sex determination and dosage compensation. The key factor in Drosophila sex determination is the female specific RNA binding protein, sex lethal (SXL) ${ }^{30}$. The maintenance of sex specific $S x l$ expression is based on autoregulatory alternative splicing that leads to the production of functional SXL proteins in females and a truncated, inactive SXL in males. However, the initiation of $S x l$ expression is primarily a transcriptional response, which is regulated by the expression levels of specific X-encoded proteins that serve to relay the dose of the $\mathrm{X}$ chromosome ${ }^{30}$ and also influenced by local chromatin environment of the female specific early $S x l_{P e}$ promoter $^{31}$. In males in the absence of SXL the Male Specific Lethal (MSL) complex is formed and dosage compensation is activated ${ }^{32}$. MSL increases the transcriptional output from the single $\mathrm{X}$ chromosome by acetylation of lysine 16 on histone H4. Loss of dosage compensation in males and its activation in females are both lead to lethality ${ }^{32}$. Thus, both sex determination and dosage compensation are influenced by chromatin structure and thus, might be influenced by the availability of histones, such as His4r. However, whether loss of His4r leads to more pronounced female sublethality by disturbing these processes is yet to be determined. We also investigated the effects of loss of His4r on adult phenotypes like daily activity or aging but we observed no remarkable defects. These data together suggest that the functions of His4r are mostly but not fully redundant and might be substituted by $\mathrm{H} 4$ or the processes it is involved in are not essential under standard environmental conditions.

As a histone protein His4r is expected to have a role in chromatin organization. During the $\mathrm{S}$ phase of the cell cycle the large amounts of histones required for chromatin replication are supplied by replication-dependent genes of the histone gene cluster. However, histones are also deposited to chromatin during the interphase. The histone $\mathrm{H} 3.3$ variant is known to be incorporated into chromatin in a replication independent manner and to replace $\mathrm{H} 3$ on transcriptionally active genes ${ }^{33,34}$ and also at silenced heterochromatic regions ${ }^{35}$ by the histone regulator A (HIRA) ${ }^{36}$ and death-associated protein (DAXX)/ $\alpha$-thalassemia X-linked mental retardation protein $(\text { ATRX })^{35}$ histone chaperon complexes, respectively. In mice, this process leads to the accumulation of H3.3 at near saturation levels ( $~ 99 \%$ of total H3) in liver, kidney and brain cells by late adulthood ${ }^{37}$. As the HIRA and DAXX/ATRX histone chaperone complexes bind $\mathrm{H} 3.3-\mathrm{H} 4$ dimers ${ }^{38}$ histone replacement requires equimolar amounts of $\mathrm{H} 3.3$ and $\mathrm{H} 4$. We assume that His4r as a replication independent histone is a partner of $\mathrm{H} 3.3$ in this process and might have similar effects on chromatin organization and transcription. To investigate these assumptions, we performed position-effect variegation (PEV) and transcriptomic analysis. Our data show that partial loss of His $4 r$ acts as a mild PEV suppressor, suggesting that it contributes to the formation or maintenance of condensed chromatin structure. Importantly, loss of function mutations of DLP, the Drosophila homolog of the heterochromatic H3.3-H4 specific histone chaperone DAXX, also suppress PEV ${ }^{35}$. In transcriptomic analysis we found that only a small subset of genes showed significantly altered transcriptional activity in $H_{i s} 4 r^{\Delta 42}$ male flies and this set was not enriched for genes specific for any biological process. The general trend of gene expression changes in the absence of His4r (67 genes up-regulated and 34 genes down-regulated) was similar to what was observed by others in the absence of H3.3 (288 up- and 99 down-regulated genes) ${ }^{28}$ i.e. in accordance with their role in chromatin formation up-regulation of transcriptional activity was more common in the absence of either His4r or H3.3.

As the loss of His $4 r$ has no remarkable adult phenotypes and only mild effects on transcription under standard conditions we decided to test its potential role in environmental stress response. For this we chose to analyze the response to heat shock that is commonly used in chromatin dynamics studies. Upon heat stress Heat Shock Factor (HSF), the Drosophila homologue of the mammalian heat stress responsive transcriptional factor HSF1, binds to its recognition sequence (Heat Shock sequence Elements, HSE) in trimeric form and elicits complex transcription regulatory steps ${ }^{39}$. The presence of HSEs is not sufficient to HSF binding in vivo, however, it is also highly dependent on the local chromatin environment. Genome-wide ChIP-seq analysis showed that HSF was present at less than $15 \%$ of HSE elements in the genome after heat shock and it co-localized with marks of active chromatin including acetylated $\mathrm{H} 3$ and $\mathrm{H} 4$, trimethylated $\mathrm{H} 3 \mathrm{~K} 4$, dimethylated $\mathrm{H} 3 \mathrm{~K} 79$ and mono-ubiquitylated $\mathrm{H} 2 \mathrm{~B}^{40}$. Heat shock protein (HSP) genes whose products function as molecular chaperones are well characterized 
targets of HSF. The molecular events of HSP activation in flies are described in most detail in the case of $H s p 70$ and $H s p 90$ genes. Upon heat shock HSF releases RNA polymerase II from promoter-proximal pausing with the help of transcription elongation factors and in interaction with nucleosome remodeling factors and histone modifying enzymes reorganizes chromatin by inducing $\mathrm{H} 2 \mathrm{~K} 5$ and $\mathrm{H} 4$ specific acetylation and ADP ribosylation of histones and eviction of nucleosomes ${ }^{39,41}$. Consequently, active transcription results in the disassembly of nucleosomes on HSP genes. After heat shock, on nucleosome depleted Hsp70 genes H3.3 histone variant containing nucleosomes are assembled with the aid of XNP chromatin remodeler and HIRA and ASF1 histone chaperones ${ }^{36}$.

We performed heat shock experiments with His $4 r$ mutant adults and found that the survival rate of young His $4 r^{\Delta 42}$ flies is higher compared to the control upon $37^{\circ} \mathrm{C}$ heat shock treatment. $34^{\circ} \mathrm{C}$ preconditioning increased the survival rate of control flies to the level observed in the His $4 r$ mutant strain without preconditioning but it did not cause notable change in the survival rate of His $4 r$ mutants. Furthermore, in contrast to the significantly improved stress resistance that we observed in the case of young adults the survival rate of 1-month-old His $4 r^{\Delta 42}$ flies was only slightly better compared to the control, which probably results from the loss of His $4 r$ with age as based on our data its transcriptional activity gradually declines during aging. These results suggest that the higher survival rate of $\mathrm{His}_{4} \mathrm{r}^{\Delta 4}$ flies after heat shock might be the consequence of a less condensed chromatin structure in the absence of His4r that can be also achieved by preconditioning or aging. Preconditioning and older age abolishes the positive effects of loss of His $4 r$ on heat stress tolerance because on the one hand preconditioning might lead to the restructuring of chromatin while on the other hand in older adults the supply of available His $4 \mathrm{r}$ is limited.

We measured the transcriptional activity of different HSP genes to ascertain whether altered HSP expression coincides with the above described effects in the lack of His4r. Heat shock treatment at $37^{\circ} \mathrm{C}$ provoked increased induction of Hsp27 and Hsp68 in the absence of His4r that might be responsible for the higher survival rate of $H i s 4 r^{\Delta 42}$ flies. However, preconditioning at $34^{\circ} \mathrm{C}$ abolished the difference between His $4 r$ mutant and control flies by raising HSP gene expression levels of the controls to the levels observed in $H i s 4 r^{\Delta 42}$ flies, while causing no notable transcriptional change in the His $4 r^{\Delta 42}$ strain itself. In conclusion, the increased survival rate of young $H i s 4 r^{\Delta 42}$ flies after heat shock goes hand in hand with stronger induction of some HSP genes. However, preconditioning and old age cancels these effects suggesting that preconditioning, age and the absence of His4r might exert similar effects on local chromatin structure.

To further confirm our hypothesis we performed chromatin accessibility assays both at the regulatory region and at the gene body of the Hsp27 gene in heat shock experiments with or without preconditioning. In the Hsp27 promoter three HSEs are located between -370 and -270 upstream of the transcriptional start site ${ }^{26}$. The sequence between the TATA box and the HSEs are organized by a positioned nucleosome ${ }^{42}$ and is dispensable for heat induction in vitro ${ }^{26}$. Our results show that heat shock induces nucleosome remobilization both at the HSE containing regulatory region and at the gene body of Hsp27, however, loss of His $4 r$ only affects chromatin accessibility at the HSEs. Although upon heat shock chromatin is more accessible at the Hsp27 promoter in the absence of His4r, preconditioning cancels this effect which is in concordance with increased post-heat shock survival and HSP gene expression induction. Interestingly, our results indicate that chromatin accessibility at the gene body is not affected by His4r as no difference was observed between His $4 r$ mutant and control flies.

In conclusion, our findings suggest that although His4r is a non-essential protein in fruit flies it might play a role in fine tuning the transcriptional response to environmental stress conditions by participating in the dynamic formation of local chromatin structure at regulatory regions of inducible genes.

\section{Materials and methods}

Fly stocks and crosses. Fly stocks were maintained and crosses were done on standard Drosophila medium at $25^{\circ} \mathrm{C}$ unless otherwise noted. The $y^{1} w^{67 c 23} ; P\{E P g y 2\} H i s 4 r^{E Y 06726}$ line was from the Bloomington Drosophila Stock Center, $y^{*} w^{*} ; P\{w[+m W \cdot h s]=F R T(w[h s])\} 2 A \quad P\{r y[+t 7.2]=n e o F R T\} 82 B$ PBac $\{$ SAstopDsRed $\} L L 05512$ $P\{y[+t 7.7] r y[+t 7.2]=$ Car20y $\} 96 E / T M 6 B, T b^{1}$ was from the Kyoto Stock Center (DGRC).

To generate His $4 r$ deletion lines $y^{1} w^{67 c 23} ; P\{E P g y 2\} H i s 4 r^{E Y 06726}$ males were mated with $w$; Dr $\Delta 2-3 / T M 3 \Delta 2-3$ females, then $w$; $P\{E P g y 2\} H i s 4 r^{E Y 06726} / T M 3 \Delta 2-3$ male jumpstarter F1 offspring were crossed with $w$; TM2/TM6 females. $w$; His $4 r^{\text {revertant } / T M 2 ~ r e v e r t a n t ~ m a l e ~ F 2 ~ p r o g e n y ~ w e r e ~ s e l e c t e d ~ b a s e d ~ o n ~ t h e ~ w h i t e ~ e y e ~ c o l o r ~ a p p e a r i n g ~}$ as a result of the loss of the $\mathrm{P}\{\mathrm{EPgy} 2\}$ element and crossed individually to $w$; TM2/TM6 females. Five days after mating founder $w$; His $4 r^{\text {revertant } / T M 2 ~ F 2 ~ m a l e s ~ w e r e ~ r e m o v e d ~ f r o m ~ t h e ~ v i a l s ~ a n d ~ w e r e ~ g e n o t y p e d ~ b y ~ P C R ~ w h i l e ~}$ their progenies were used to establish $w$; His $4^{\text {revertant }}$ lines.

Deletion analysis by PCR. Individual w; His4revertant/TM2 male candidates were homogenized in $50 \mu \mathrm{l}$ squashing buffer ( $10 \mathrm{mM}$ Tris- $\mathrm{HCl}, 1 \mathrm{mM}$ EDTA, $25 \mathrm{mM} \mathrm{NaCl}, 200 \mathrm{ng} / \mu$ l Proteinase $\mathrm{K}$ ) and incubated for $60 \mathrm{~min}$ at $37^{\circ} \mathrm{C}$ followed by $15 \mathrm{~min}$ at $85^{\circ} \mathrm{C} .4 \mu \mathrm{l}$ homogenates were used as templates in $20 \mu \mathrm{l}$ PCR reactions using DreamTaq DNA polymerase (Thermo Fisher Scientific, TFS) with His4rFseq forward and His4rgR or His4rE3CR (Supplementary Table S1) reverse primers at $250 \mathrm{nM}$ concentration. His4r deletions were identified based on the presence of PCR products shorter than with wild-type control template. Sanger capillary sequencing was performed on purified His4rFseq-His4rgR PCR amplicons with His4rFseq sequencing primer by Delta Bio 2000 Ltd. (Szeged, Hungary).

Complementation test and fecundity assay. $\quad w$; His $4 r^{\Delta 42}$ (henceforth $H i s 4 r^{\Delta 42}$ ) virgins were mated to $y^{*} w^{*} ; P\{w[+m W . h s]=F R T(w[h s])\} 2 A \quad P\{r y[+t 7.2]=n e o F R T\} 82 B \quad P B a c\{S A s t o p D s R e d\} L L 05512 P\{y[+t 7.7]$ $r y[+t 7.2]=$ Car20y $\} 96 E / T M 6 B, T b^{1}$ (henceforth $\left.H_{i s} 4 r^{L L 05512}\right)^{20}$ males to generate $w$; His $4 r^{L L 5512} / H i s 4 r \quad{ }^{\Delta 42}$ progeny. w; His $4 r^{L L 05512} / H i s 4 r^{\Delta 42}$ males and females were crossed separately with $w^{1118}$ flies then the vials were checked for the presence of viable progeny. 
To measure the fecundity of homozygous His $4 r^{\Delta 42}$ and His $4^{\text {rev5 }}$ flies freshy eclosed (0-24 h old) males and females were collected and mated for 3 days on yeast rich medium before the experiment. 10 parallel crosses were made per genotype, with five females and five males in each vial. Flies were passed to a fresh vial every day for 5 days and the number of eggs laid were counted in each vial. Fecundity is expressed as the average number of eggs laid per female per day \pm standard error of mean (SEM).

Viability assays. To determine the eclosion rate of $H i s 4 r^{442}$ flies a two-step crossing scheme was performed. Homozygous His $4 r^{\Delta 42}$ virgins were mated with $M\{3 x P 3-R F P$.attP $\} Z H-86 F b$ males (a homozygous viable strain commonly used as a docking site for $\varphi \mathrm{C} 31$ integrase mediated transgenesis, henceforth called $86 \mathrm{Fb}$ ), then heterozygous $\mathrm{His}_{4} r^{\Delta 42} / 86 \mathrm{Fb}$ males were backcrossed to $\mathrm{His}_{4} r^{\Delta 42}$ virgins in 30 parallel vials. The number of homozygous and heterozygous progeny was determined based on the presence of the RFP marker. Data is shown as the total number of eclosed flies, for statistical analysis binomial test and Student's t-test were used.

To determine the ratio of male and female progeny during the third larval stage freshly eclosed $\mathrm{His}_{4} \mathrm{r}^{42}$ or $H i s 4 r^{\text {rev } 5}$ males and females were mated for 3 days on yeast rich medium, then sorted to 20 vials ( 5 males and 5 females each) per genotype. Wandering 3 rd instar larvae (wL3) were collected and sorted under a stereo microscope where male and female larvae can be distinguished by their gonads. Sorted larvae were placed on fresh vials where pupation and eclosion analysis was carried out. Data is shown as mean \pm standard error of mean (SEM), for statistical analysis Student's t-test was used.

Longevity assay. Freshly eclosed His $4 r^{\text {rev5 }}$ and His $4 r^{\Delta 42}$ males and females were collected separately (30 flies/ vial). To determine longevity, flies were kept at $25^{\circ} \mathrm{C}$, transferred to fresh vials every second day and the number of deceased individuals was recorded daily. At least 240 flies per genotype were used for longevity analysis. For statistical analysis OASIS 2 (Online Application for Survival Analysis 2) software was used ${ }^{43}$.

Daily activity measurement. Freshly eclosed His $4 r^{\text {rev5 }}$ and $H i s 4 r^{442}$ males were collected and kept at $25^{\circ} \mathrm{C}$ (30 flies/vial). Flies were synchronized and entrained by exposing them to 12:12 h light ( 250 lx):dark (LD) cycles for 1 week before performing the activity recordings with DAM2 Drosophila Activity Monitor (TriKinetics Inc, Waltham, MA, USA) that records the activity of 32 individual flies simultaneously. We recorded the daily activity of flies over a period of $24 \mathrm{~h}$ starting from ZT0 (Zeitgeber Time: refers to time in hours during a lightdark cycle where ZT0 = lights on and ZT12=lights off). Data were collected with DAMSystem3 for Windows and analyzed using pySolo analysis software ${ }^{24}$ and by Excel functions. Data are presented as mean \pm standard error of mean (SEM).

PEV analysis. His $4 r^{r e v 5}$ and His $4 r^{\Delta 42}$ males were mated with $\operatorname{In}(1) \mathrm{w}^{\mathrm{m}}{ }^{4 \mathrm{~h}}$ females (in which a chromosomal inversion places the white gene next to the pericentric heterochromatin ${ }^{44}$ ) to produce progeny. Variegation of the white gene was quantitated by measuring eye pigment content. To extract eye pigments 20 fly heads were homogenized in $1.2 \mathrm{ml}$ volume of $1: 1$ mixture of chloroform and $0.1 \%$ ammonium-hydroxide. After phase separation by centrifugation at $13 \mathrm{kRPM}$ for $3 \mathrm{~min}$ the upper water phase containing the pigments was collected and its optical density was measured at $485 \mathrm{~nm}$ in a spectrophotometer. Data are presented as mean \pm standard error of mean (SEM), for statistical analysis Student's t-test was used.

RNA-sequencing and data analysis. Total RNA was prepared from 7-day-old adult males (3 biological replicates per genotype, 30 males per replicate) using Trizol Reagent (Invitrogen) and MN NucleoSpin RNA Clean-up kit (Macherey-Nagel). RNA integrity and concentration were determined with Agilent 2100 Bioanalyzer capillary gel electrophoresis using Agilent RNA 6000 Nano kit. Poly(A) RNA fraction from 800 ng total RNA was selected with NEBNext Poly(A) mRNA Magnetic Isolation Module (New England Biolabs, NEB) then strand-specific, indexed RNA-sequencing libraries were prepared using NEBNext Ultra Directional RNA Library Prep Kit for Illumina (NEB). Fragment length distribution and concentration of sequencing libraries were determined with Agilent 2100 Bioanalyzer using Agilent DNA 1000 kit, then after pooling and denaturing libraries were sequenced in an Illumina MiSeq DNA sequencer using MiSeq Reagent Kit V3-150. Paired-end sequence reads were inspected with FastQC then quality trimmed using Trimmomatic v0.33 with options HEADCROP:15 CROP:55 ILLUMINACLIP:TruSeq3-PE.fa:2:30:10:8:true TRAILING:10 SLIDINGWINDOW:4:20 MINLEN:36. Trimmed read pairs were aligned to the Drosophila melanogaster reference genome Dmr6.13 using TopHat v2.0.9 spliced read mapper. After sorting and deduplicating binary alignment files with Samtools 0.1.19, differential gene expression analysis was done with Cuffdiff v2.1.1 using Drosophila gene annotation release r6.13.gtf. For gene ontology term analysis we applied PANTHER Overrepresentation Test (released 20190711) with GO Ontology database (released 2019-10-08) using Fisher's exact test with FDR correction.

Heat shock response analysis. Heat shock-response analysis was carried out on both young (3-5 daysold) and on old (1-month-old) His $4 r^{r e v 5}$ and His $4 r^{\Delta 42}$ males and females (20 flies per vial, 30 parallel vials per genotype) reared at $25^{\circ} \mathrm{C}$. Young flies were exposed to $37^{\circ} \mathrm{C}$ heat shock for $0 \mathrm{~min}$ (no-hs controls), $1 \mathrm{~h}, 2 \mathrm{~h}$, $3 \mathrm{~h}, 3.5 \mathrm{~h}, 4 \mathrm{~h}, 4.5 \mathrm{~h}$, or $5 \mathrm{~h}$ while old flies were exposed to $37^{\circ} \mathrm{C}$ heat shock for 0 min (no-hs controls), $15 \mathrm{~min}$, $30 \mathrm{~min}, 45 \mathrm{~min}, 1 \mathrm{~h}, 1 \mathrm{~h} 15 \mathrm{~min}, 1 \mathrm{~h} 30 \mathrm{~min}, 1 \mathrm{~h} 45 \mathrm{~min}$, or $2 \mathrm{~h}$. After heat shock flies were transferred to fresh vials and the number of survivors was counted the next morning. Preconditioning of flies was carried out by daily heat shocks at $34^{\circ} \mathrm{C}$ for $30 \mathrm{~min}$ for two consecutive days prior to the $37^{\circ} \mathrm{C}$ heat shock treatment. Data are presented as mean \pm standard error of mean (SEM), for statistical analysis OASIS 2 (Online Application for Survival Analysis 2) software and Mann-Whitney U test were used. 
Gene expression analysis by RT-QPCR. For validation of proper gene expression in $H i s 4 r^{\text {rev } 5}$ and loss of expression in His $4 r^{\Delta 42}$ flies 7-days-old Oregon-R, His $4 r^{\text {rev5 }}$ and His $4 r^{\Delta 42}$ males were collected.

For analysis of age dependent His $4 r$ expression freshly eclosed $w^{1118}$ wild-type male flies were collected (30 flies/ vial) and kept at $25^{\circ} \mathrm{C}$, sampling times were the following: 1 day, 3 days, $1,2,4,6$ and 8 weeks.

For analysis of HSP gene induction 3-5 days-old His $4 r^{\text {rev }}$ and $\mathrm{His}_{4} \mathrm{r}^{\Delta 4}$ males were exposed to $1 \mathrm{~h} 37^{\circ} \mathrm{C}$ heat shock treatment. Samples were collected before treatment and at $0 \mathrm{~min}, 30 \mathrm{~min}, 1 \mathrm{~h}, 1.5 \mathrm{~h}, 2 \mathrm{~h}, 3 \mathrm{~h}$ and $4 \mathrm{~h}$ post-treatment. Preconditioning of flies was carried out at $34^{\circ} \mathrm{C}$ for $30 \mathrm{~min}$ for two consecutive days prior to heat shock treatment.

Total RNA was isolated from heads (at least 3 biological replicates per sampling time, 20 males per replicate) using Trizol Reagent (Invitrogen). RNA concentration and purity were determined by spectrophotometric measurement with NanoDrop ND-1000 instrument. First-strand cDNA was prepared from 400 ng total RNA after DNaseI (TFS) treatment using TaqMan Reverse Transcription Reagents (TFS) with random hexamer primers following the recommendations of the manufacturer. The resulting CDNA was diluted 1:5 and used for qPCR by the SYBR green method with Luminaris Color HiGreen qPCR Master Mix in a PikoReal Real-Time PCR System (TFS). qPCR based expression data gained with His4r, Hsp27, Hsp60A, Hsp68 and Hsp83 gene specific primers (Supplementary Table S1) were normalized to the expression level of $\alpha$-Tubulin at 84B (CG1913) ${ }^{45}$ housekeeping gene (Supplementary Table S1). Data are presented as mean \pm standard error of mean (SEM). For statistical analysis of His4r measurements One-Way ANOVA (OWA) with Tukey HSD post-hoc test was performed, while Kruskal-Wallis test and Mann-Whitney-U test was used in case of HSP genes.

Chromatin accessibility assay. Isolation of nuclei. Chromatin accessibility assays were carried out on 3-5 days-old His $4 r^{\text {rev5 }}$ and His $4 r^{\Delta 42}$ flies. Flies were exposed to $1 \mathrm{~h} 37^{\circ} \mathrm{C}$ heat shock treatment. Samples were collected before treatment and at $0 \mathrm{~h}, 1 \mathrm{~h}$, and $4 \mathrm{~h}$ post-treatment. Nuclei were isolated from at least 3 biological replicates per sampling time, $\sim 500$ flies per replicate. Flies were frozen in liquid nitrogen, then after vortexing and separation by sieving heads and bodies were collected and pulverized in liquid nitrogen using a mortar and pestle. Once liquid nitrogen had evaporated the homogenate was transferred to a beaker containing $5 \mathrm{ml}$ ice cold buffer A + NP (60 mM KCl, 15 mM NaCl, 15 mM HEPES pH7.6, 1 mM EDTA, 0.1 mM EGTA, with freshly added $0.15 \mathrm{mM}$ spermine, $0.5 \mathrm{mM}$ spermidine, $0.5 \mathrm{mM}$ DTT, $0.5 \% \mathrm{NP}-40$ and $12 \mathrm{mM}$ EDTA) and incubated on ice for 5 min with gentle stirring. The suspension was then transferred to a Dounce homogenizer (Wheaton) and disrupted with 20 strokes of a teflon pestle. The homogenate was filtered through 2 layers of Miracloth (Merck) into a $50 \mathrm{ml}$ centrifuge tube. The pellet was scraped from the Miracloth, homogenized in $3 \mathrm{ml} \mathrm{A}+\mathrm{NP}$ buffer and filtered through the same Miracloth. $2 \mathrm{ml}$ buffer AS $(60 \mathrm{mM} \mathrm{KCl}, 15 \mathrm{mM} \mathrm{NaCl}, 1 \mathrm{mM}$ EDTA, $0.1 \mathrm{mM}$ EGTA with freshly added $0.15 \mathrm{mM}$ spermine, $0.5 \mathrm{mM}$ spermidine, $0.5 \mathrm{mM}$ DTT and $0.3 \mathrm{M}$ saccharose) was added to the filtrate, which was then centrifuged at $4{ }^{\circ} \mathrm{C}, 3000 \mathrm{rpm}$ for $5 \mathrm{~min}$. The pellet was resuspended in $5 \mathrm{ml}$ buffer A + NP, homogenized with 10 strokes in the Dounce homogenizer, then $2 \mathrm{ml}$ AS buffer was added and the suspension was centrifuged at $4{ }^{\circ} \mathrm{C}, 3000 \mathrm{rpm}$ for $5 \mathrm{~min}$. The pellet was resuspended in $5 \mathrm{ml}$ buffer A $(60 \mathrm{mM} \mathrm{KCl}$, $15 \mathrm{mM} \mathrm{NaCl}, 1 \mathrm{mM}$ EDTA, $0.1 \mathrm{mM}$ EGTA, $15 \mathrm{mM}$ Tris-HCl pH7.4 with freshly added $0.15 \mathrm{mM}$ spermine, $0.5 \mathrm{mM}$ spermidine and $0.5 \mathrm{mM} \mathrm{DTT})$, homogenized with 10 strokes in the Dounce homogenizer and centrifuged at $4{ }^{\circ} \mathrm{C}, 3000 \mathrm{rpm}$ for $5 \mathrm{~min}$. The pellet was resuspended in $500 \mu \mathrm{l}$ buffer $\mathrm{A}+0.1 \% \mathrm{NP}(60 \mathrm{mM} \mathrm{KCl}, 15 \mathrm{mM}$ HEPES pH7.6, $1 \mathrm{mM}$ EDTA, $0.1 \mathrm{mM}$ EGTA and 0.1\% NP-40). $100 \mu \mathrm{l}$ sample was set aside for concentration measurement. To crosslink chromatin $1 \mathrm{ml}$ buffer $\mathrm{A}+0.1 \% \mathrm{NP}$ and $50 \mu \mathrm{l} 30 \%$ formaldehyde was added to the remaining $400 \mu \mathrm{l}$ homogenate and incubated at room temperature while shaking at $500 \mathrm{rpm}$ for $10 \mathrm{~min}$. To stop the reaction $150 \mu \mathrm{l} 1 \mathrm{M}$ glycine was added and the homogenate was centrifuged at $4{ }^{\circ} \mathrm{C}, 3000 \mathrm{rpm}$ for $5 \mathrm{~min}$. The pellet was resuspended in $5 \mathrm{ml}$ buffer $\mathrm{A}+0.1 \% \mathrm{NP}$ then centrifuged at $4{ }^{\circ} \mathrm{C}, 3000 \mathrm{rpm}$ for $5 \mathrm{~min}$. The pellet was resuspended in $400 \mu \mathrm{l}$ buffer $\mathrm{A}+0.1 \% \mathrm{NP}$ and stored at $-80^{\circ} \mathrm{C}$. To determine chromatin concentration the $100 \mu \mathrm{l}$ sample that was set aside before crosslinking was centrifuged at $4{ }^{\circ} \mathrm{C}, 3000 \mathrm{rpm}$ for $5 \mathrm{~min}$, then the pellet was resuspended in $160 \mu \mathrm{l}$ Nuclear Lysis Buffer $(50 \mathrm{mM}$ Tris-HCl pH 8.0, 1\% SDS, $10 \mathrm{mM}$ EDTA) and DNA concentration was measured using Qubit dsDNA HS Assay Kit (TFS).

Micrococcal nuclease (MNase) digestion. Nuclei stored in buffer A $+0.1 \%$ NP were centrifuged at $3000 \mathrm{rpm}$ for $5 \mathrm{~min}$ and resuspended in TE buffer. Nuclear suspension containing $1.5 \mu \mathrm{g}$ DNA was digested with MNase (TFS) in a $20 \mu \mathrm{l}$ enzyme reaction mix containing $0.02 \mathrm{U}$ MNase at $37^{\circ} \mathrm{C}$ for 30 min with shaking at $500 \mathrm{rpm}$. After addition of $10 \mathrm{mM}$ EDTA and $100 \mu \mathrm{g} / \mathrm{ml}$ RNase A (TFS) samples were further incubated at $37^{\circ} \mathrm{C}$ with shaking at $500 \mathrm{rpm}$ for $30 \mathrm{~min}$. Reverse crosslinking of samples was done at $65^{\circ} \mathrm{C}$ with shaking at $500 \mathrm{rpm}$ overnight, then $1 \mathrm{mg} / \mathrm{ml}$ Proteinase $\mathrm{K}$ (TFS) was added and samples were incubated at $37^{\circ} \mathrm{C}$ with shaking at $500 \mathrm{rpm}$ for $2 \mathrm{~h}$.

qPCR analysis. Primer pairs were designed to the $5^{\prime} \mathrm{HSEs}^{26}$ and the coding sequence of the Hsp27 gene (Supplementary Table S1). Digested chromatin was diluted 1:10 and used for qPCR by the SYBR green method with Luminaris Color HiGreen qPCR Master Mix (TFS) in a PikoReal Real-Time PCR System (TFS). qPCR data were normalized to the undigested samples. For statistical analysis of expression values Student's t-test was performed.

\section{Data availability}

All relevant data are within the manuscript and its Supporting Information files. RNA-Seq data are deposited to the National Center for Biotechnology Information Sequence Read Archive (NCBI SRA) under accession PRJNA635790. Other datasets used and/or analyzed during the current study are available from the corresponding author on reasonable request. 
Received: 7 September 2020; Accepted: 16 February 2021

Published online: 01 March 2021

\section{References}

1. Kornberg, R. D. Chromatin structure: A repeating unit of histones and DNA. Science 184, 868-871 (1974).

2. Kornberg, R. D. Structure of Chromatin. Ann. Rev. Biochem. 46, 931-954 (1977).

3. Luger, K. Crystal structure of the nucleosome core particle at $2.8 \AA$ resolution. Nature 389, 10 (1997).

4. Babu, A. \& Verma, R. S. Chromosome structure: Euchromatin and heterochromatin. Int. Rev. Cytol. 108, 1-60 (1987).

5. Ehrenhofer-Murray, A. E. Chromatin dynamics at DNA replication, transcription and repair. Eur. J. Biochem. 271, 2335-2349 (2004).

6. Talbert, P. B. \& Henikoff, S. Histone variants: Ancient wrap artists of the epigenome. Nat. Rev. Mol. Cell Biol. 11, 264-275 (2010).

7. Schümperli, D. Cell-cycle regulation of histone gene expression. Cell 45, 471-472 (1986).

8. Schümperli, D. Multilevel regulation of replication-dependent histone genes. Trends Genet. 4, 187-191 (1988),

9. Thatcher, T. H. \& Gorovsky, M. A. Phylogenetic analysis of the core histones H2A, H2B, H3, and H4. Nucleic Acids Res. 22, 174-179 (1994).

10. Akhmanova, A., Miedema, K. \& Hennig, W. Identification and characterization of the Drosophila histone H4 replacement gene. FEBS Lett. 388, 219-222 (1996).

11. Yamamoto, Y. \& Watanabe, T. Epigenetics evolution and replacement histones: evolutionary changes at Drosophila H4r. J. Phylogenet. Evol. Biol. 4, 3 (2016).

12. van Daal, A., White, E. M., Gorovsky, M. A. \& Elgin, C. R. Drosophila has a single copy of the gene encoding a highly conserved histone H2A variant of the H2A F/Z type. Nucleic Acids Res. 16, 7487-7497 (1988).

13. Akhmanova, A. S. et al. Structure and expression of histone H3.3 genes in Drosophila melanogaster and Drosophila hydei. Genome 38, 586-600 (1995).

14. Brown, J. B. et al. Diversity and dynamics of the Drosophila transcriptome. Nature 512, 393-399 (2014).

15. Graveley, B. R. et al. The developmental transcriptome of Drosophila melanogaster. Nature 471, 473-479 (2011).

16. Siegel, T. N. et al. Four histone variants mark the boundaries of polycistronic transcription units in Trypanosoma brucei. Genes Dev. 23, 1063-1076 (2009).

17. Hays, S. M., Swanson, J. \& Selker, E. U. Identification and characterization of the genes encoding the core histones and histone variants of Neurospora crassa. Genetics 160(3), 961-973 (2002).

18. Gendron, N., Dumont, M., Gagne, M.-F. \& Lemaire, S. Poly A-containing histone H4 mRNA variant žH4-v.1/: Isolation and sequence determination from bovine adrenal medulla. Biochim. Biophys. Acta 1396(1), 32-38 (1998).

19. Schuldiner, O. et al. piggyBac-Based mosaic screen identifies a postmitotic function for cohesin in regulating developmental axon pruning. Dev. Cell 14, 227-238 (2008).

20. McKay, D. J. et al. Interrogating the function of metazoan histones using engineered gene clusters. Dev. Cell 32, 373-386 (2015).

21. Nakahata, Y., Grimaldi, B., Sahar, S., Hirayama, J. \& Sassone-Corsi, P. Signaling to the circadian clock: Plasticity by chromatin remodeling. Curr. Opin. Cell Biol. 19, 230-237 (2007).

22. Grimaldi, B., Nakahata, Y., Kaluzova, M., Masubuchi, S. \& Sassone-Corsi, P. Chromatin remodeling, metabolism and circadian clocks: The interplay of CLOCK and SIRT1. Int. J. Biochem. Cell Biol. 41, 81-86 (2009).

23. Ripperger, J. A. \& Schibler, U. Rhythmic CLOCK-BMAL1 binding to multiple E-box motifs drives circadian Dbp transcription and chromatin transitions. Nat. Genet. 38, 369-374 (2006).

24. Gilestro, G. F. Video tracking and analysis of sleep in Drosophila melanogaster. Nat. Protoc. 7, 995-1007 (2012).

25. Elgin, S. C. R. \& Reuter, G. Position-effect variegation, heterochromatin formation, and gene silencing in Drosophila. Cold Spring Harb. Perspect. Biol. 5, a017780 (2013).

26. Riddihough, G. \& Pelham, H. R. B. Activation of the Drosophila hsp27 promoter by heat shock and by ecdysone involves independent and remote regulatory sequences. EMBO J. 5, 1653-1658 (1986).

27. Talbert, P. B. et al. A unified phylogeny-based nomenclature for histone variants. Epigenet. Chromatin 5, 7 (2012).

28. Sakai, A., Schwartz, B. E., Goldstein, S. \& Ahmad, K. Transcriptional and developmental functions of the H3.3 histone variant in Drosophila. Curr. Biol. 19, 1816-1820 (2009)

29. Hödl, M. \& Basler, K. Transcription in the absence of Histone H3.3. Curr. Biol. 19, 1221-1226 (2009).

30. Salz, H. \& Erickson, J. W. Sex determination in Drosophila: The view from the top. Fly 4, 60-70 (2010).

31. Rodriguez, J. \& Horabin, J. I. Polycomb/Trithorax group proteins collaborate with Heterochromatin protein 1 to regulate Drosophila sex determination. Epigenet. Chromatin 6, P70 (2013).

32. Lucchesi, J. C. \& Kuroda, M. I. Dosage compensation in Drosophila. Cold Spring Harb. Perspect. Biol. 7, a019398 (2015).

33. Ahmad, K. \& Henikoff, S. The histone variant H3.3 marks active chromatin by replication-independent nucleosome assembly. Mol. Cell 9, 1191-1200 (2002).

34. Wirbelauer, C. Variant histone H3.3 is deposited at sites of nucleosomal displacement throughout transcribed genes while active histone modifications show a promoter-proximal bias. Genes Dev. 19, 1761-1766 (2005).

35. Fromental-Ramain, C., Ramain, P. \& Hamiche, A. The Drosophila DAXX-like protein (DLP) cooperates with ASF1 for H3.3 deposition and heterochromatin formation. Mol. Cell. Biol. 37, e00597 (2017).

36. Schneiderman, J. I., Orsi, G. A., Hughes, K. T., Loppin, B. \& Ahmad, K. Nucleosome-depleted chromatin gaps recruit assembly factors for the H3.3 histone variant. Proc. Natl. Acad. Sci. USA 109, 19721-19726 (2012).

37. Tvardovskiy, A., Schwämmle, V., Kempf, S. J., Rogowska-Wrzesinska, A. \& Jensen, O. N. Accumulation of histone variant H3.3 with age is associated with profound changes in the histone methylation landscape. Nucleic Acids Res. 45, 9272-9289 (2017).

38. Hammond, C. M., Strømme, C. B., Huang, H., Patel, D. J. \& Groth, A. Histone chaperone networks shaping chromatin function. Nat. Rev. Mol. Cell Biol. 18, 141-158 (2017).

39. Guertin, M. J., Petesch, S. J., Zobeck, K. L., Min, I. M. \& Lis, J. T. Drosophila heat shock system as a general model to investigate transcriptional regulation. Cold Spring Harb. Symp. Quant. Biol. 75, 1-9 (2010).

40. Guertin, M. J. \& Lis, J. T. Chromatin landscape dictates HSF binding to target DNA elements. PLoS Genet. 6, e1001114 (2010).

41. Miozzo, F., Sabéran-Djoneidi, D. \& Mezger, V. HSFs, stress sensors and sculptors of transcription compartments and epigenetic landscapes. J. Mol. Biol. 427, 3793-3816 (2015).

42. Quivy, J.-P. \& Becker, P. B. The architecture of the heat-inducible Drosophila hsp27Promoter in nuclei. J. Mol. Biol. 256, 249-263 (1996).

43. Han, S. K. et al. OASIS 2: online application for survival analysis 2 with features for the analysis of maximal lifespan and healthspan in aging research. Oncotarget 7, 56147-56152 (2016).

44. Vogel, M. J. et al. High-resolution mapping of heterochromatin redistribution in a Drosophila position-effect variegation model. Epigenet. Chromatin 2, 1 (2009).

45. Ponton, F., Chapuis, M.-P., Pernice, M., Sword, G. A. \& Simpson, S. J. Evaluation of potential reference genes for reverse transcription-qPCR studies of physiological responses in Drosophila melanogaster. J. Insect Physiol. 57, 840-850 (2011). 


\section{Acknowledgements}

We thank Dr. Nóra Zsindely for useful discussions and Zita Kóra for technical assistance. Stocks obtained from the Bloomington Drosophila Stock Center were used in this study. László Bodai was supported by the Hungarian National Research, Development and Innovation Office (NKFIH) grants K-112294, GINOP-2.3.2-15-201600032 and GINOP-2.3.2-15-2016-00034, by the UNKP-20-5-SZTE-642 New National Excellence Program of the Ministry for Innovation and Technology and by the János Bolyai Research Scholarship (BO/00522/19/8) of the Hungarian Academy of Sciences. Anikó Faragó was supported by the NTP-NFTÖ-18-B-0359 National Youth Excellence Scholarship and by the UNNP-19-3-SZTE-72 New National Excellence Program of the Ministry for Innovation and Technology. The APC was funded by University of Szeged Open Access Fund grant 4953. The funders had no role in study design, data collection and analysis, decision to publish, or preparation of the manuscript.

\section{Author contributions}

A.Farag. participated in study design, acquisition, analysis and interpretation of data and drafting the manuscript. A.Ü. and A.Fark. participated in acquisition and interpretation of data and revising the manuscript. L.B. participated in study conception and design of experiments, statistical analysis and interpretation of data and revising the manuscript. All authors read and approved the final manuscript.

\section{Competing interests}

The authors declare no competing interests.

\section{Additional information}

Supplementary Information The online version contains supplementary material available at https://doi. org/10.1038/s41598-021-84413-4.

Correspondence and requests for materials should be addressed to L.B.

Reprints and permissions information is available at www.nature.com/reprints.

Publisher's note Springer Nature remains neutral with regard to jurisdictional claims in published maps and institutional affiliations.

(c) (i) Open Access This article is licensed under a Creative Commons Attribution 4.0 International License, which permits use, sharing, adaptation, distribution and reproduction in any medium or format, as long as you give appropriate credit to the original author(s) and the source, provide a link to the Creative Commons licence, and indicate if changes were made. The images or other third party material in this article are included in the article's Creative Commons licence, unless indicated otherwise in a credit line to the material. If material is not included in the article's Creative Commons licence and your intended use is not permitted by statutory regulation or exceeds the permitted use, you will need to obtain permission directly from the copyright holder. To view a copy of this licence, visit http://creativecommons.org/licenses/by/4.0/.

(C) The Author(s) 2021 